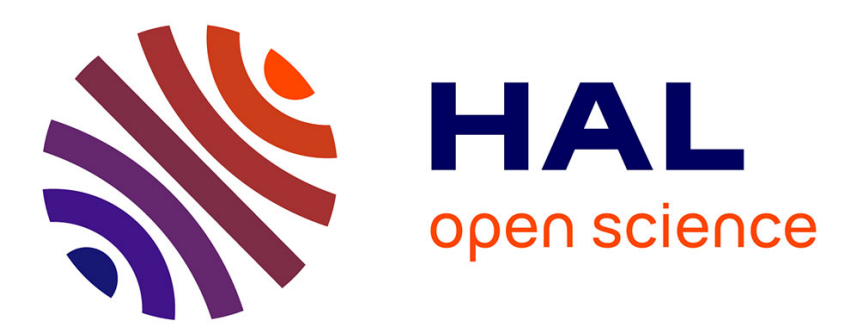

\title{
Carrières de marbre des Pyrénées centrales : le point sur la recherche
}

Jean-Marc Fabre, Robert Sablayrolles

\section{To cite this version:}

Jean-Marc Fabre, Robert Sablayrolles. Carrières de marbre des Pyrénées centrales: le point sur la recherche. Gallia - Archéologie de la France antique, 2002, Carrières antiques de la Gaule, 59, pp.61-81. 10.3406/galia.2002.3097 . hal-01770317

\section{HAL Id: hal-01770317 https://hal.science/hal-01770317}

Submitted on 18 Apr 2018

HAL is a multi-disciplinary open access archive for the deposit and dissemination of scientific research documents, whether they are published or not. The documents may come from teaching and research institutions in France or abroad, or from public or private research centers.
L'archive ouverte pluridisciplinaire HAL, est destinée au dépôt et à la diffusion de documents scientifiques de niveau recherche, publiés ou non, émanant des établissements d'enseignement et de recherche français ou étrangers, des laboratoires publics ou privés.

\section{(ㅇ)(1) $\$$}

Distributed under a Creative Commons Attribution - NonCommercial - NoDerivatives| 4.0 


\title{
Carrières de marbre des Pyrénées centrales. Le point sur la
} recherche

\author{
In: Gallia. Tome 59, 2002. pp. 61-81.
}

\section{Résumé}

Les études récentes sur les carrières de marbre des Pyrénées se sont orientées dans trois directions : un travail de terrain (prospection et sondages) à des fins d'inventaire et d'établissement d'une chronologie fiable fondée sur des données assurées ; une recherche scientifique de critères d'identification des matériaux; une analyse de la diffusion à partir de lots de mobilier issus de grands sites régionaux. Les travaux récents sur les objets en marbre typiques des vallées pyrénéennes (autels votifs et auges cinéraires) permettent de mesurer l'impact de l'économie du marbre sur la vie et la culture des populations montagnardes de l'Antiquité.

\section{Abstract}

The recent studies on ancient Pyrenean marble quarries turned towards three directions : field work (prospecting and excavations) to make a survey and fix up a chronology based on reliable data ; search of scientific identification criteria for the materials ; analysis of circulation based on series of archaeological remains found in major regional sites. Recent research on typical marble remains from the Pyrenean valleys (votive altars and cinerary urns) helps to measure the impact of marble economy on life and culture of the mountain people in Antiquity.

Citer ce document / Cite this document :

Fabre Jean-Marc, Sablayrolles Robert. Carrières de marbre des Pyrénées centrales. Le point sur la recherche. In: Gallia. Tome 59, 2002. pp. 61-81.

doi : 10.3406/galia.2002.3097

http://www.persee.fr/web/revues/home/prescript/article/galia_0016-4119_2002_num_59_1_3097 


\title{
CARRIÈRES DE MARBRE DES PyRÉNÉES CENTRALES
}

\author{
Le point sur la recherche
}

\author{
Jean-Marc FABre et Robert SABLAYROLLES
}

Mots-clés. Pyrénées, marbre, griolle, archéométrie, aulels, auges, acculturalion.

Key-words. Pyreneas, marble, griotte, archaeometry, allars, urns, culture.

\begin{abstract}
Résumé. Les études récentes sur les carrières de marbre des Pyrénées se sont orientées dans trois directions : un travail de terrain prospection et sondages) à des fins d'inventaire et d'établissement d'une chronologie fiable fondée sur des données assurées; une recherche. cientifique de critères d'identification des matériaux; une analyse de la diffusion à partir de lots de mobilier issus de grands sites rígionaux. Les travaux récents sur les objets en marbre typiques des vallées pyrénéennes (autels votifs et auges cinéraires) permettent de mesurer l'impact de l'économie du marbre sur la vie et la culture des populations montagnardes de l'Antiquité.
\end{abstract}

Aostract. The recent studies on ancient Byrenean marble quarries turned towards three directions : field work (prospecting and ox cavations) to make a survey and fix up a chronology based on reliable data; search of scientific identification criteria for the materials; analysis of circulation based on series of archaeological remains found in major regional sites. Recent research on lypical marble remains from the Pyrenean valleys (volive altars and cinerary urns) helps to measure the impact of marble economy on life and culture of the mountain people in Antiquity.

L'évolution récente de l'archéologie, dans ses techniques comme dans ses problématiques, a radicalement changé les perspectives d'analyse: le temps n'est plus - ou ne devrait plus être, il existe de regrettables exceptions - aux vastes synthèses globalisantes et définitives. Le souci du détail et de l'exhaustivité dans l'étude de terrain repousse la synthèse à la constitution d'une base de données, cohérente et fiable, et la spécificité des approches, suivant les domaines, rend délicate la maitrise de matières souvent diverses et variées. La présente contribution se veut donc un état des recherches, qui montre autant le chemin à parcourir que les étapes déjà franchies et ne saurait, en aucune manière, épuiser la question de l'exploitation du marbre dans les Pyrénées centrales. Elle s'appuie sur un travail de longue haleine, qui débuta par des prospections-inventaires (donc très générales) du territoire de la cité des Convènes, et notamment du haut bassin de la Garonne, situé en amont de la capitale, Lugdunum, l'actuelle bourgade de Saint-Bertrand-de-Comminges (Fabre, 1991; Saunière, 1992 ; Sablayrolles, 2001). Ces travaux, qui mirent en évidence le rôle prépondérant joué par l'extraction et la commercialisation des marbres dans le haut bassin de la Garonne, furent suivis de prospections thématiques, spécifiquement orientées sur les sites potentiels d'extraction antique (Fabre, 1999b; Fabre, Lucas, 2001). Cette démarche s'accompagna d'un inventaire et d'une étude de collections disponibles sur des sites particulièrement riches de la région proche ou plus lointaine, comme la cité antique de Lugdunum, les villae de Valentine et 
Montmaurin, l'agglomération secondaire de Cosa, au nord de la ville actuelle de Montauban, ou quelques villae rurales de la même région. Enfin, des travaux d'archéométrie furent menés parallèlement à cette double approche du terrain et des collections, de façon à chercher des critères de caractérisation, qui permettent d'établir un lien plus assuré entre les échantillons des carrières et les éléments de mobilier découverts sur les sites archéologiques (Costedoat, 1992, 1995 ; Fabre, Lucas, 2001). Cette enquête, qui allie investigations de terrain, études de collections et recherches archéométriques, s'inscrit dans une tradition déjà ancienne, jalonnée par les écrits pionniers de F. Braemer (1969) et les colloques plus récents, organisés en 1995 à SaintBertrand-de-Comminges, sur la question de la caractérisation des marbres blancs (Cabanot et al. éds, 1995), et en 1999 (table ronde), à Toulouse, sur l'exploitation des ressources naturelles des Pyrénées durant l'Antiquité (Sablayrolles dir., 2001). Ce survol rapide de l'état des recherches et de la bibliographie disponible souligne les limites de l'entreprise. Les recherches de terrain paraissent, pour l'heure, avoir épuisé le potentiel disponible dans les bassins des Pyrénées centrales (Adour, Garonne et Salat) ct une suite éventuelle n'est, dans ce domaine, envisagcable que dans le cadre de découverte fortuite sur des sites d'extraction actuels, où l'exploitation moderne, par le remaniement de déblais anciens, ferait ressurgir, comme ce fut le cas, en 1946, à la carrière de Rapp, près de Saint-Béat, des traces d'exploitation antique ${ }^{36}$. Les analyses de collections, en revanche, peuvent et doivent être complétées et la recherche archéométrique n'est pas, elle non plus, arrivée à épuisement de ses possibilités. L'analyse des travaux de terrain, des essais de caractérisation et des études de mobilier présentée ici se veut donc un reflet ponctuel, géographiquement comme chronologiquement, de ce que nous savons de l'exploitation antique du marbre dans les Pyrénées centrales. Le caractère provisoire du bilan n'interdit pas d'ouvrir des perspectives historiques, même s'il faut éviter le piège,

36. Les fouilles menées à l'époque (Sapène, 1946), avant la destruction du site par l'exploitation moderne, mirent en évidence, au-dessus du sol de carrière proprement dit, l'existence d'un atelier de taille puis d'un sanctuaire visiblement lić à l'exploitation marbrière. La stratigraphic, l'étude du mobilier et de l'épigraphie ont donné lieu à une réévaluation récente (Fabre, Sablayrolles, 1995). Depuis, seule, la petite carrière du Pujo de (íry, près de Saint-Béat, a fait l'objet d'unc exploration archéologique (Fabre, 1999a). dénoncé en commençant, de la synthèse prématurée, hâtive, trop large et mal étayée. L'influence de l'économie du marbre (artisanat ou industrie ?) dans la vallée de la Garonne est suffisamment manifeste, mesurée à l'aune de ce mobilier original et de ces pratiques métissées que constituèrent localement autels votifs et auges cinéraires, pour que l'on puisse esquisser les lignes des transformations économiques, sociales, politiques et culturelles qu'induisirent, dans le haut bassin antique de la Garonne, l'exploitation et la commercialisation du marbre.

\section{LES DONNÉES DE TERRAIN}

Dans le cadre d'un programme de prospection thématique (Service régional de l'archéologie de MidiPyrénées 1995-1999) ${ }^{37}$, un premier travail de repérage et d'échantillonnage des gisements a été effectué; sur certains sites, le travail de prospection, qui avait donné quelques indices chronologiques anciens, a été prolongé par des sondages. La zone géographique étudiée, les Pyrénées centrales, comprend la partie méridionale de trois départements: l'Ariège, la Haute-Garonne et les Hautes-Pyrénées (fig. 44 à 46). Les gisements marbriers sont situés au nord de la haute chaîne axiale, que suit approximativement la frontière franco-espagnole. De chaque côté de la faille nord-pyrénéenne, qui a joué un rôle important dans la genèse des roches métamorphiques, on trouve une concentration des principaux matériaux utilisés dans l'Antiquité, c'est-à-dire les brèches, les griottes et les marbres blancs ou gris.

\section{LES BRÈCHES}

Plusieurs types de brèches ont été exploités dans la région, mais, dans l'Antiquité, c'est la Brèche Isabelle, composée d'éléments de calcite blanche et d'un ciment de couleur jaune, orange, parfois verdâtre, qui a connu le

\footnotetext{
37. Voir Fabre, 1999b. Ce programme, intégré à un projet collectif de recherche sur l'exploitation des ressources naturelles dans les Pyrénées centrales durant l'Antiquité, dirigé par R. Sablayrolles, a donné lieu à une table ronde en octobre 1999 (Sablayrolles dir., 2001). Les informations concernant le travail de terrain et les études de mobilier reprennent dans ses grandes lignes la communication présentée lors de cette table ronde (Fabre, lucas, 2001).
} 


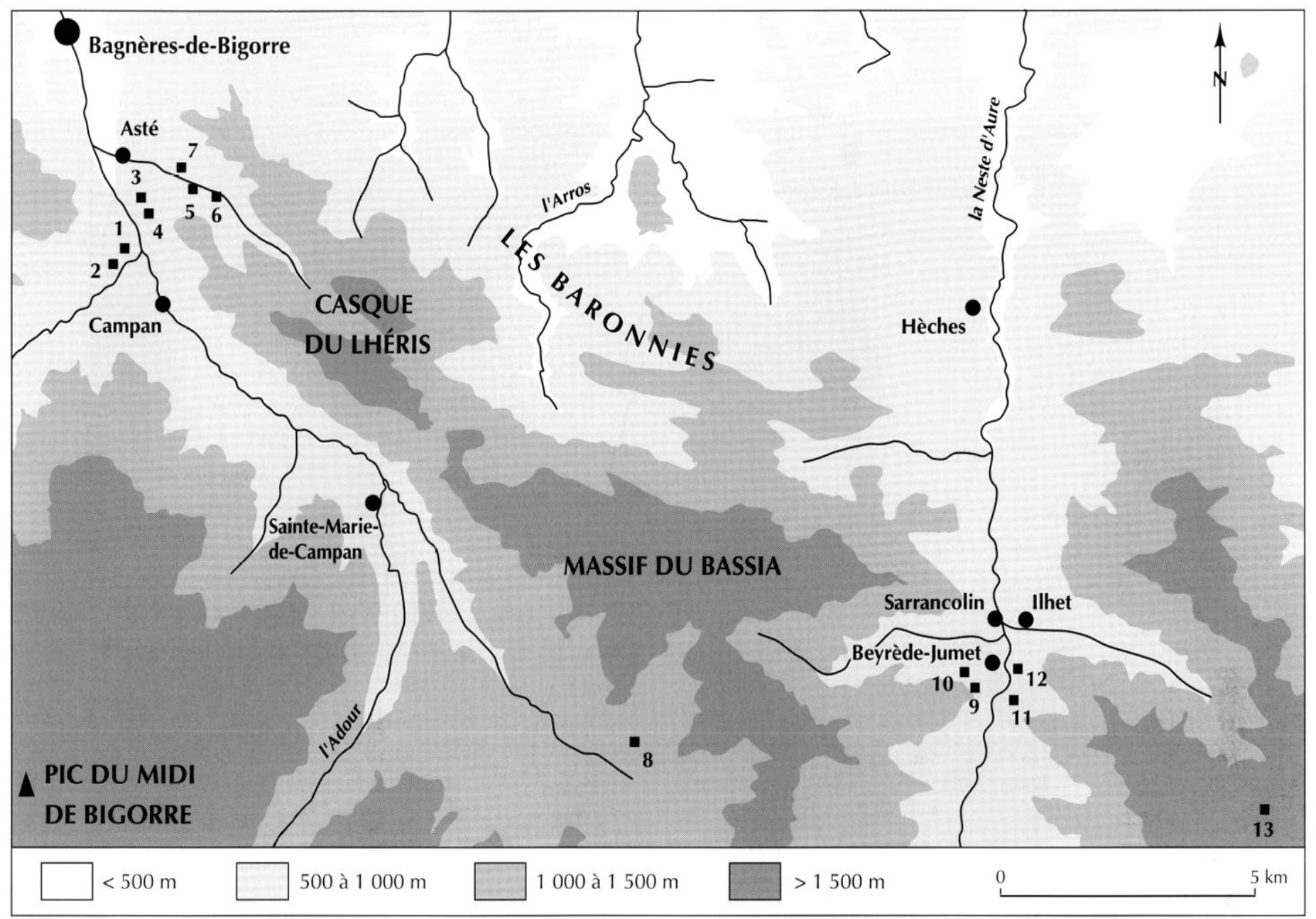

Fig. 44 - Carte de localisation des gisements marbriers des vallées d'Aure el de l'Adlour duns les Hautes-Pyrénées : 1, 2, Beaudéan, carrières de marbre gris ; 3, 4, Asté, carrières de brèche et de marbre gris ; 5, 6, Asté, carrières de brèche ; 7, Asté, carrière de marbre gris; 8, Sainte-Mariede-Campan, carrière de griolle de L'Lspiadet ; 9, 10, Beyrède-Jumet, carrières de brèche multicolore; 11, Ilhet, carrière de brèche multicolore de L'Fscolle; 12, Ilhet, affleurement brèchique de Chagarde; 13, Nistos, affleurement de brèche de Cap Nestès (DAO J.-M. Fabre, CNRS).

plus grand succès. Cette brèche tectonique a été exploitée sur deux gisements, à Asté (fig. 44, $\mathrm{n}^{\text {Ns }} 5$ et 6 ), et à La Pène-Saint-Martin, près de Saint-Béat, sur le versant méridional du Cap del Mount (fig. 45, $\mathrm{n}^{\circ}$ 16), mais les vestiges visibles sont pour la plupart du XIX $\mathrm{X}^{\mathrm{e}}$ ou du début du $\mathrm{XX}^{\mathrm{e}} \mathrm{s}$. Seule la partie supérieure du front de carrière de Saint-Béat peut être rattachée à la phase d'exploitation antique ${ }^{38}$ (fig. 47). Les autres matériaux, notamment les brèches multicolores de la vallée d'Aure (fig. 44, nos 9, 10 , 11), n'ont probablement pas été exploités durant

38. Au XIX" s., la réouverture de cette carrière a entrainé la destruction d'un cimetière médiéval, auquel fait référence le toponyme, qui fournissait un terminus ante quem pour l'exploitation située au-dessus. Ce matériau ne semble pas avoir été utilisé durant le Moyen Âge (Fabre et al., 1995, p. 122-123).
l'Antiquité ${ }^{39}$; l'utilisation importante de ces marbres, dits de Sarrancolin, dans les bâtiments royaux à l'époque moderne est à l'origine de leur renommée actuelle (Bernigole, 1995 ; Bresc-Bautier, 1995).

\section{LES GRIOTTES}

Calcaires du Dévonien, caractérisés par une grande variété de structures et de teintes, du vert au rouge, ces matériaux sont communément dénommés marbre de

39. À l'heure actuelle, sur plus de 5000 fragments de placages antiques inventoriés dans la région, aucun matériau provenant des gisements de la vallée d'Aure n'a été relevé. 


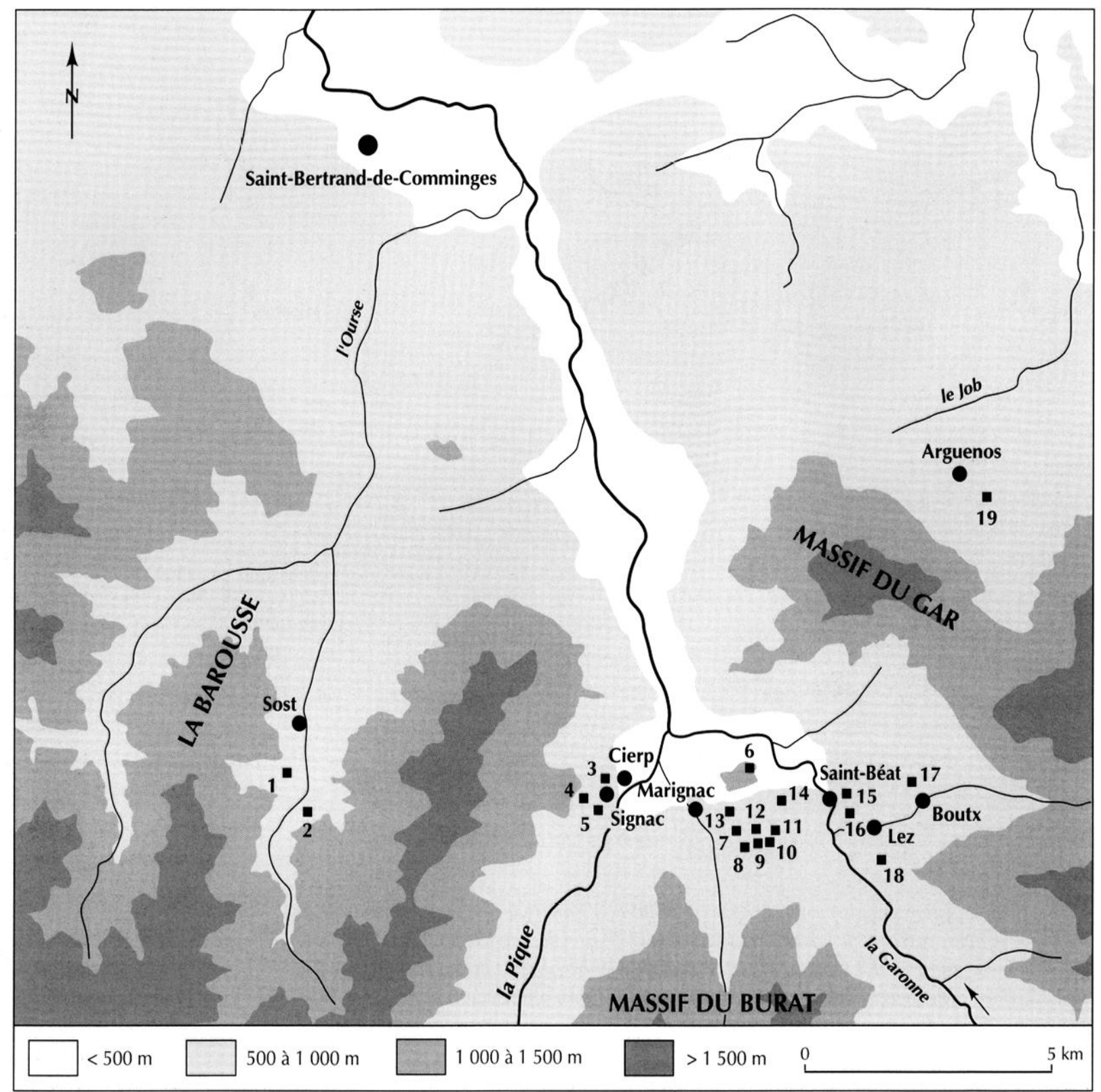

Fig. 45 - Carte de localisation des gisements marbriers de la vallée de la Garonne : 1, Sost, carrière de marbre blanc au Cap de la Lane; 2, Sost, carrière de griotte rouge ; 3, Cierp-Gaud, carrière de griotte rouge; 4, Signac, carrière de griotte rouge de La Goule; 5, Signac, carrière de griotte rouge et vert de Lepaux ; 6, Saint-Béat, carrière de marbre blanc du Pujo de Géry; 7, Marignac, carrière de marbre blanc de Saint-Martin ; 8, Marignac, carrière de griotte de Mailh Blanc 3; 9, Marignac, carrière de griotte à dominante rouge de Mailh Blanc 2; 10, Marignac, carrière de griotte de Mailh Blanc 1; 11, Marignac, carrière de calcaire ; 12, 13, Marignac, carrières de marbre blanc; 14, Saint-Béat, carrière de Rapp ; 15, Saint-Béal, carrière du château ; 16, Saint-Béat, carrière de La Pène-Saint-Martin ou brèche romaine; 17, Boutx, carrière de marbre blanc de La Pène ; 18, Lez, carrière de griotte à dominante verte ; 19, Arguenos, carrière de marbre blanc (DAO J.-M. Fabre, CNRS).

Campan ou cipollino mandolato. D'est en ouest, au sud de la faille, ils affleurent en de très nombreux points des Pyrénées centrales, mais on peut distinguer principalement trois zones de production.

Dans la vallée de l'Adour, le gisement de L'Espiadet, commune de Sainte-Marie-de-Campan (fig. $44, \mathrm{n}^{\circ} 8$ ), présente plusieurs types de griottes, dont les plus caractéristiques sont très colorés, rouge et vert, avec de grandes veines de calcite blanche. L'exploitation moderne, continue depuis le XVII ${ }^{e}$ s., semble avoir détruit tous les fronts de carrière antérieurs, mais l'exploitation antique est cependant attestée par l'utili- 
sation courante de ce matériau sur les sites antiques du sud-ouest de la Gaule.

Dans la vallée de la Garonne, pas moins de huit carrières ont été recensées et tous les faciès de griottes sont représentés sur les communes de Sost, Signac, Cierp-Gaud, Marignac, Saint-Béat et Lez. Dans cette large gamme de produits, on peut opposer les griottes rouge lie-de-vin de Sost, peu métamorphisées, à certaines griottes aux teintes très pâles de Lez, ou aux matériaux beaucoup plus métamorphisés de Mailh Blanc, où la structure amygdalaire n'apparaît pratiquement plus. Dans le bassin de la Garonne, le contexte archéologique local est particulièrement riche, mais les parcours de terrain n'ont pu mettre en évidence des vestiges d'exploitation antique des griottes. Ici encore, seule l'étude des éléments de marbre découverts sur les sites archéologiques, avec le problème crucial de la caractérisation du matériau, est susceptible de donner quelques indications sur cette zone de production.

Le problème est encore plus délicat pour les carrières de griotte du Haut-Salat, Ariège (fig. $46, \mathrm{n}^{\mathrm{s}} 3,4,5$ ), où le contexte archéologique régional apparaît relativement pauvre. $\mathrm{Au} \mathrm{XX} \mathrm{XX}^{\mathrm{e}}$ s., l'exploitation a détruit les éventuels vestiges anciens, et l'identification du matériau reste le seul lien entre gisement et site archéologique. Les griottes de l'Ariège sont des matériaux homogènes, aux bonnes qualités esthétiques et techniques; la structure amygdalaire, avec des nodules parfois très allongés, est soulignée par des contrastes de couleur importants dans la gamme des roses/rouges ou des verts.

Dans l'ensemble, hormis quelques rares exemples particulièrement typiques des gisements, comme celui de L'Espiadet près de Sainte-Marie-de-Campan, il est cependant difficile d'affirmer la provenance des matériaux utilisés sur les sites gallo-romains.

\section{LES MARBRES BLANCS OU GRIS}

Les marbres blancs, blanc et gris ou gris ont été exploités dans de multiples carrières sur toute la zone étudiée. Cependant, tous les indices archéologiques mis au jour proviennent du secteur de Saint-Béat, qui concentre par ailleurs les seules exploitations concernant des volumes importants. Hors de cette zone, la prospection n'a mis au jour aucun vestige d'exploitation ancienne (fig. $44, \mathrm{n}^{\text {os }} 1,2,3,7$; fig. $45, \mathrm{n}^{\mathrm{os}} 1,19$; fig. 46 , $\mathrm{n}^{\circ} 6$ ), et la plupart des carrières semblent relativement récentes, le matériau ayant bien souvent servi comme pierre à bâtir dans la région.

Dans la haute vallée de la Garonne, deux sites ont livré des vestiges d'exploitation antique: le Pujo de Géry (fig. 45, $\mathrm{n}^{\circ} 6$ ) et la Montagne d'Arri (fig. 45, $\left.\mathrm{n}^{\circ} 14\right)$. Pour le premier, il s'agit d'un petit site d'extraction, où les carriers se sont contentés de tirer quelques blocs de grande taille (environ $1 \mathrm{~m} \times 2 \mathrm{~m} \times 0,5 \mathrm{~m}$ ), tout au plus une dizaine. Le plan d'extraction horizontal a été fouillé puis relevé, et les environs minutieusement prospectés et sondés par endroits, pour mettre au jour une éventuelle extension (Fabre, 1999a). Sur les aspects techniques et sur le plan de l'organisation de la production, on ne peut considérer ce site comme représentatif de l'exploitation marbrière locale; tout au plus témoigne-t-il de la coexistence de petits sites d'extraction au voisinage d'exploitations beaucoup plus importantes, comme celle de la Montagne d'Arri, située à $150 \mathrm{~m}$ au sud.

À Saint-Béat, comme à Sarrancolin en vallée d'Aure, la vallée de la Garonne, qui pénètre au sein de la zone métamorphique, devient très étroite et le relief particulièrement abrupt. Le village est enserré entre deux montagnes de marbre culminant à $1200 \mathrm{~m}$, le Cap del Mount et la Montagne d'Arri, dont les versants septentrionaux ont été largement exploités depuis l'Antiquité. La permanence de cette activité importante a détruit la plus grande partie des vestiges antiques et les seuls témoins éventuellement conservés sont masqués par des tonnes de déblais. Dans un tel contexte, la prospection classique ne donne guère de résultats et il faut donc surveiller les travaux pour lesquels l'exploitant est parfois contraint de déplacer des déblais; cependant, et malheureusement, l'exploitation actuelle se développe principalement en souterrain. En 1946, c'est un éboulement qui avait entraîné la mise au jour, dans la carrière de Rapp, d'un front de taille de la fin du ${ }^{\text {er }}$ s. avant notre ère, réutilisé en sanctuaire au II $^{\mathrm{e}} \mathrm{s}$. (Sapène, 1946 ; Fabre, Sablayrolles, 1995). Le site, dénommé Mailh de las Higouras, a été détruit peu après sa découverte.

\section{AUTRES MATÉRIAUX}

Dans les Pyrénées centrales, l'abondance des marbres semble avoir exclu l'utilisation d'autres roches égale- 


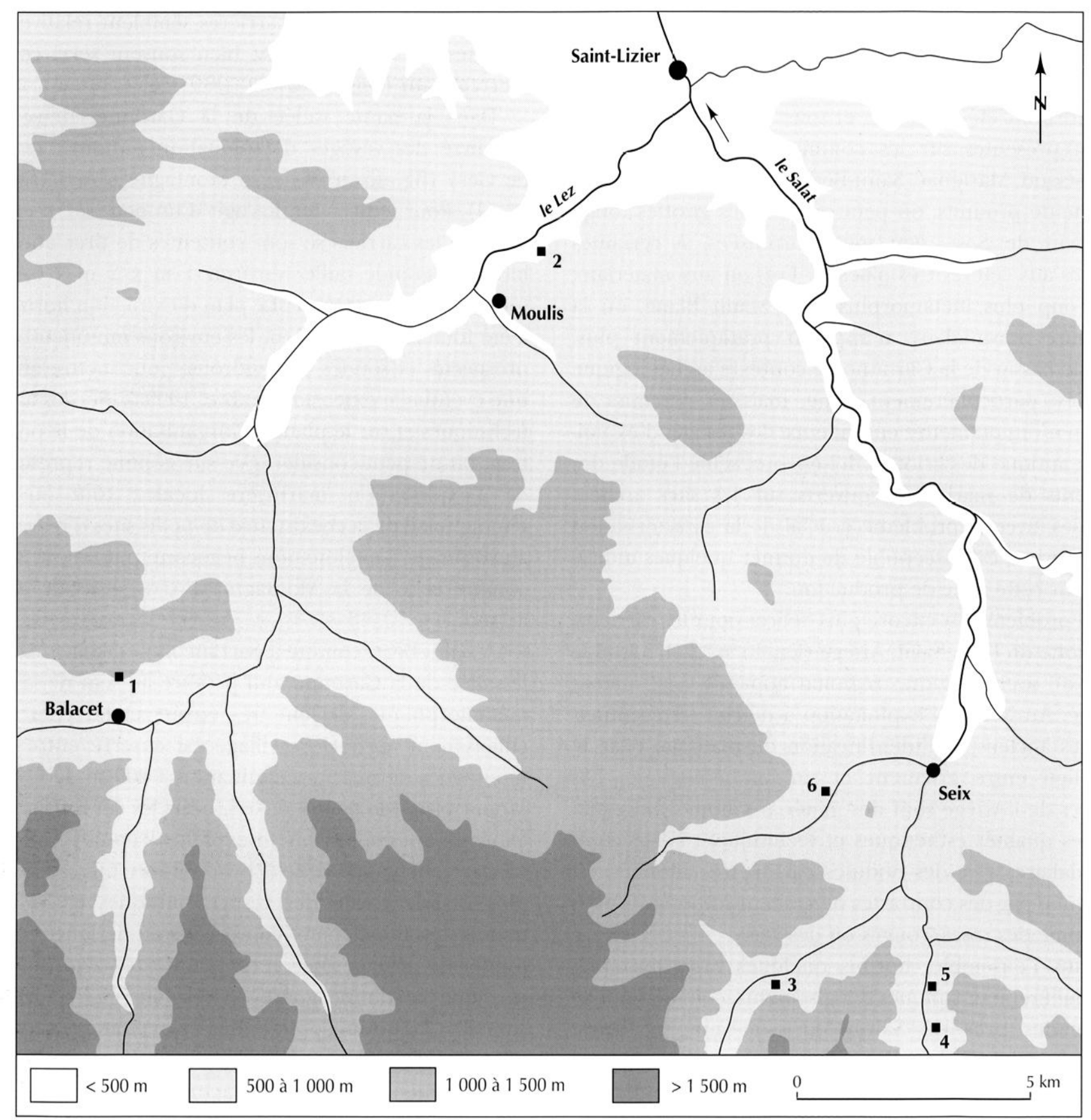

Fig. 46 - Carte de localisation des gisements marbriers de l'Ariège : 1, Balacet, carrière de brèche multicolore ; 2, Moulis, carrière d'Aubert ; 3, Seix, carrière de griotte d'sistours ; 4, Couftens, carriere de griotle de Font des Tourdets; 5, Seix, carriere de griotle d'iscalortes ; 6, Seix, carrière de martre blanc discalirous (DAOJ.-M. Fabre, CNRS).

ment disponibles, les granites par exemple, comme pierres décoratives. Les calcaires du piémont ont été réservés à la fourniture de matériaux de construction. Seul le calcaire métamorphisé noir à veines blanches caractéristiques d'Aubert, commune de Moulis (fig. 46, $\mathrm{n}^{\circ} 2$ ), a été débité en placages. Il est réputé pour avoir connu une diffusion lointaine, notamment jusqu'à
Rome ${ }^{40}$. Sur le site, hormis la présence d'une villa à proximité de la carrière, il y a peu d'indices archéologiques : l'exploitation moderne, qui s'est développée en fosse, a probablement détruit les anciens fronts de taille, et,

40. Pour l'utilisation de ce matériau à Rome, voir Gnoli, 1971, p. 168-169. 
à la périphérie, la prospection systématique et les sondages à la tarière pédologique n'ont donné aucun résultat.

\section{LA PLACE PRIVILÉGIÉE DE LA VALLÉE DE LA GARONNE}

Le bilan du travail de terrain effectué durant cinq années apparaît plutôt décevant sur le plan des vestiges d'extraction antique. Bien souvent, l'exploitation moderne au fil hélicoïdal ou au foret n'a pas épargné le moindre mètre carré de front plus ancien et l'on doit se résigner désormais à surveiller les travaux susceptibles d'exhumer des vestiges. La prospection de l'ensemble des gisements a cependant permis de constituer une collection d'échantillons de référence.

La rareté des vestiges d'exploitation n'autorise aucune conclusion définitive, mais on peut d'ores et déjà faire quelques observations générales. L'essentiel de la production de marbre antique se répartit, sans que puisse être établie de véritable hiérarchie, sur trois secteurs, la région de Saint-Béat, la vallée de l'Adour et la haute vallée du Salat. Ces zones de production sont situées dans les trois vallées principales, orientées nord-sud, qui constituent les seuls axes de pénétration au cour de la zone interne métamorphique. Cependant, l'accès depuis la plaine sous-pyrénéenne est beaucoup plus facile pour le secteur de Saint-Béat : en effet, jusqu'aux carrières, la vallée est relativement large et le dénivelé peu accentué (fig. 45). De plus, les carrières de Saint-Béat bénéficient d'un marché de proximité relativement important, avec la cité de Lugdunum, capitale des Convènes, et un dense réseau de villae dans un rayon d'environ $20 \mathrm{~km}$. Tout cela, ainsi que la variété des matériaux disponibles, a dû favoriser l'essor des sites d'extraction et des ateliers saint-béatais. Les deux autres secteurs semblent, eux, plus spécialisés dans la production des griottes, sans que l'on puisse estimer leur part dans la production d'ensemble.

Le site de Moulis n'entre pas dans cette organisation générale ; peut-être l'originalité du matériau suffit-elle à expliquer son isolement relatif ?

\section{CARACTÉRISATION DES MATÉRIAUX}

Ces dernières années, dans le sillage des grands programmes internationaux sur les marbres grecs, turcs et italiens, les scientifiques se sont intéressés aux gisements du sud de la Gaule et la connaissance des matériaux pyrénéens a beaucoup progressé. Ainsi, les marbres blancs et les griottes des Pyrénées centrales ont chacun fait l'objet d'une thèse de géologie appliquée à l'archéologie (Costedoat, 1992; Antonelli, 1997), destinée à caractériser les matériaux afin de permettre d'identifier l'origine des objets archéologiques retrouvés en fouille ou conservés dans les musées.

Ces travaux comportent tous deux une importante collecte d'échantillons, 167 griottes et 151 marbres blancs, qui constituent une base de données de référence jusque-là inédite. Chaque échantillon a fourni une ou plusieurs lames minces, et tous les matériaux, observés au microscope, ont été précisément décrits. L'étude pétrographique et minéralogique est souvent riche d'enseignements, mais ne permet pas de distinguer les différents gisements. En effet, les marbres et les calcaires plus ou moins métamorphisés ont une histoire commune, des conditions de formation souvent très proches et des éléments remarquables peu nombreux. Une analyse géochimique détaillée, portant sur les éléments traces des matériaux, ne permet pas, non plus, de caractériser les différents ensembles; les géologues doivent donc avoir recours à d'autres méthodes, plus complexes, telles que la cathodoluminescence et la géochimie isotopique. Cette dernière méthode permet d'établir des diagrammes de rëférences représentant le champ isotopique de chaque gisement. Certaines zones se recoupent ou même se superposent, telles que Gère-Bélesten, Boutx, Saint-Péde-Bigorre, Sost, Arguenos et Saint-Béat pour les marbres blancs, ou Sost, Argut-Dessous, Cierp-Gaud, Espiougue (commune d'Esplas-de-Sérou), Seix et Couflens pour les griottes, mais la combinaison des résultats avec ceux des autres méthodes permet dans certains cas de distinguer les matériaux. Pour les griottes, seule une méthode de traitement statistique des résultats chimiques et isotopiques est à même de caractériser les différents spécimens.

$\mathrm{Si}$, par le biais de ces travaux récents, la connaissance des gisements pyrénéens a considérablement progressé, on peut cependant s'interroger sur les difficultés de mise en œuvre de telles méthodes d'analyse et, surtout, sur le problème de leur application à l'archéologie.

Le premier problème concerne la représentativité de l'échantillonnage effectué. Les géologues, pour des raisons de délais et de crédits, en partie liées à leur enga- 
gement universitaire, ont été contraints de faire des choix dans les sites à étudier. Ainsi, sept carrières de marbres blancs et dix carrières de griottes ont été choisies, selon l'importance et l'ancienneté supposées de leur exploitation (Antonelli, 1997). Bien sûr, ces choix sont discutables et l'on peut se demander, par exemple, pourquoi les carrières de Signac, au cœur même du district de Saint-Béat/Marignac, n'ont pas été retenues ${ }^{41}$ ? Pour les griottes, on constate un fort déséquilibre entre l'échantillonnage effectué en Ariège (85 échantillons) et les autres zones marbrières, qui présentent pourtant un contexte archéologique beaucoup plus riche. Ainsi, le seul site d'extraction du Pont-de-la-Taule (Seix), de petite extension, totalise 42 échantillons, alors que 15 échantillons prélevés dans la carrière de Cierp-Gaud sont censés représenter plusieurs gisements des environs. La conséquence principale de cet échantillonnage partiel des gisements est l'impossibilité d'identifier la provenance d'un matériau. Tout au plus peut-on rattacher un échantillon, avec un certain degré d'incertitude, à l'un des gisements étudiés. Cette incertitude est renforcée par l'impossibilité matérielle d'échantillonner les fronts de taille antiques, aujourd'hui détruits par les exploitations modernes.

Tous ces problèmes sont bien illustrés par les tentatives d'application des méthodes d'identification sur des marbres antiques. Sur 25 échantillons de chapiteaux en marbre blanc analysés, 20 sont problématiques; 11 n'entrent dans aucun des diagrammes isotopiques prédéfinis et 9 présentent une pétrographie contradictoire avec les données isotopiques! Pour les griottes, 12 échantillons sur 45 n'entrent pas dans les diagrammes isotopiques « convenus » et $80 \%$ des échantillons n'ont pu faire l'objet d'une analyse géochimique en raison de leur taille trop réduite. De toute évidence, le protocole d'analyse est inadapté, probablement pour des contraintes techniques, et les ensembles définis pour caractériser les gisements ne satisfont pas les besoins. Il faudrait probablement augmenter le nombre d'échantillons de chaque carrière (et non adapter le diagramme isotopique aux données issues des analyses d'objets archéologiques), afin d'agrandir ou de préciser leur

41. L'auteur évoque la proximité de Cierp-Gaud, dont le gisement présente un matériau très comparable (Antonelli, 1997, p. 58). L'argument est quelque peu difficile à recevoir quand on connaît les importantes variations, notamment isotopiques, au sein d'un même gisement. domaine, et étudier tous les gisements, afin de délimiter éventuellement des nouveaux champs. Cela représente un travail important et très onéreux, qui n'est pas envisageable dans un proche avenir. À l'heure actuelle, la rigueur scientifique appelle la modestie et, plutôt que de proposer des identifications qui demeurent hypothétiques, il serait préférable d'orienter les recherches vers une évaluation des marges d'erreur, ou bien d'étudier des séries cohérentes et relativement importantes de vestiges afin de, modestement, proposer des tendances.

Dans sa thèse, C. Costedoat avait proposé un test statistique pour calculer « la probabilité de nonséparation entre un objet et une carrière ". L'exemple choisi, 4 échantillons du trophée de Saint-Bertrand-deComminges, n'était pas vraiment problématique, puisque les analyses isotopiques comme la cathodoluminescence indiquaient Saint-Béat. Ce test a été appliqué là pour tenter de préciser la provenance précise des pièces du trophée et a désigné sans ambiguité la carrière du château, sur la rive droite ; il faudrait peut-être l'appliquer de façon beaucoup plus systématique pour évaluer à chaque résultat les marges d'erreur.

Un autre développement a été testé par C. Costedoat, en étroite collaboration avec J. Cabanot (1995). L'étude porte sur 321 chapiteaux préromans conservés dans les musées aquitains. Tous les prélèvements ont fait l'objet d'une étude pétrographique, d'une analyse isotopique et d'une cathodoluminescence. Les résultats mettent en lumière deux ensembles bien distincts attribués à SaintBéat (276 pièces) et aux carrières de la vallée d'Ossau (45 pièces). Même si l'on doit rester encore prudent, le caractère systématique de cette recherche et le nombre de pièces pris en compte autorisent certaines conclusions :

- l'importance et la durée de la production des carrières de Saint-Béat ;

- la concurrence de gisements moins importants, mais uniquement sur le marché de proximité ;

- la place conséquente du travail de taille sur les chantiers de construction, au détriment de l'idée d'un monopole des ateliers situés près des carrières:

$\mathrm{Si}$, du point de vue de la méthodologie analytique, il semble nécessaire de poursuivre les recherches, cette étude alliant étroitement le travail d'un archéologue et d'un géologue semble exemplaire. Cette coopération, qui met à la disposition des sciences humaines des techniques d'analyses sophistiquées, est indispensable. Le 
travail d'Antonelli, victime du cloisonnement scientifique et universitaire, souffre de quelques approximations archéologiques et historiques. Aussi débouchet-il sur des conclusions d'ordre historique un peu rapides, qui peuvent paraître décalées par rapport aux problématiques actuelles de la recherche en sciences humaines ${ }^{42}$

\section{PRODUCTION ET DIFFUSION DES ATELIERS PYRÉNÉENS}

\section{DONNÉES CONTRASTÉES DES MARCHÉS RÉGIONAUX}

La présentation des différentes méthodes d'analyse utilisées montre les difficultés d'une caractérisation scientifique des matériaux, et il est peut-être trop tôt pour affirmer la provenance précise d'un marbre pyrénéen. En revanche, dans bien des cas, on peut distinguer les matériaux pyrénéens des marbres importés. Cela est relativement facile pour les marbres colorés, brèches du type de La Pène-Saint-Martin (fig. 47) ou griottes, et pour les marbres blancs à passes grises, à gros cristaux, typiques des Pyrénées centrales et de la région de SaintBéat. C'est par contre plus aléatoire pour les marbres blancs, et, dans l'examen des lots de placages antiques, nous avons considéré que seuls les marbres blancs découverts dans les sites archéologiques de la région de production, à 15-20 km maximum des carrières, étaient pyrénéens. Au-delà, les marbres blancs sont considérés comme de "provenance indéterminée ".

Les lots de marbres étudiés proviennent des fouilles de la villa et de l'ensemble cultuel tardifs de Valentine ${ }^{43}$, de la domus de Coupéré, à Saint-Bertrand-deComminges ${ }^{44}$, et de ramassages de surface sur des

42. Tout le problème réside dans la représentativité des échantillons analysés. Même si l'on accepte les identifications proposées, on ne peut en aucun cas tirer des conclusions sur la production des gisements pyrénéens à partir de quelques " sondages " effectués en France et en Italie. Que représente un fragment de plaquette de griotte verte conservée à Ostie à côté des mètres cubes de marbres pyrénéens utilisés sur les sites antiques proches des carrières, qui, eux, n'ont pas été échantillonnés, comme Montmaurin, Valentine ou Lugdunum des Convènes, site sur lequel on a prélevé seulement deux échantillons dans un bâtiment médiéval (!) ?

43. Fouilles de G. Fouet, entre 1957 et 1981

44. Fouilles de R. Sablayrolles, entre 1995 et 1998.

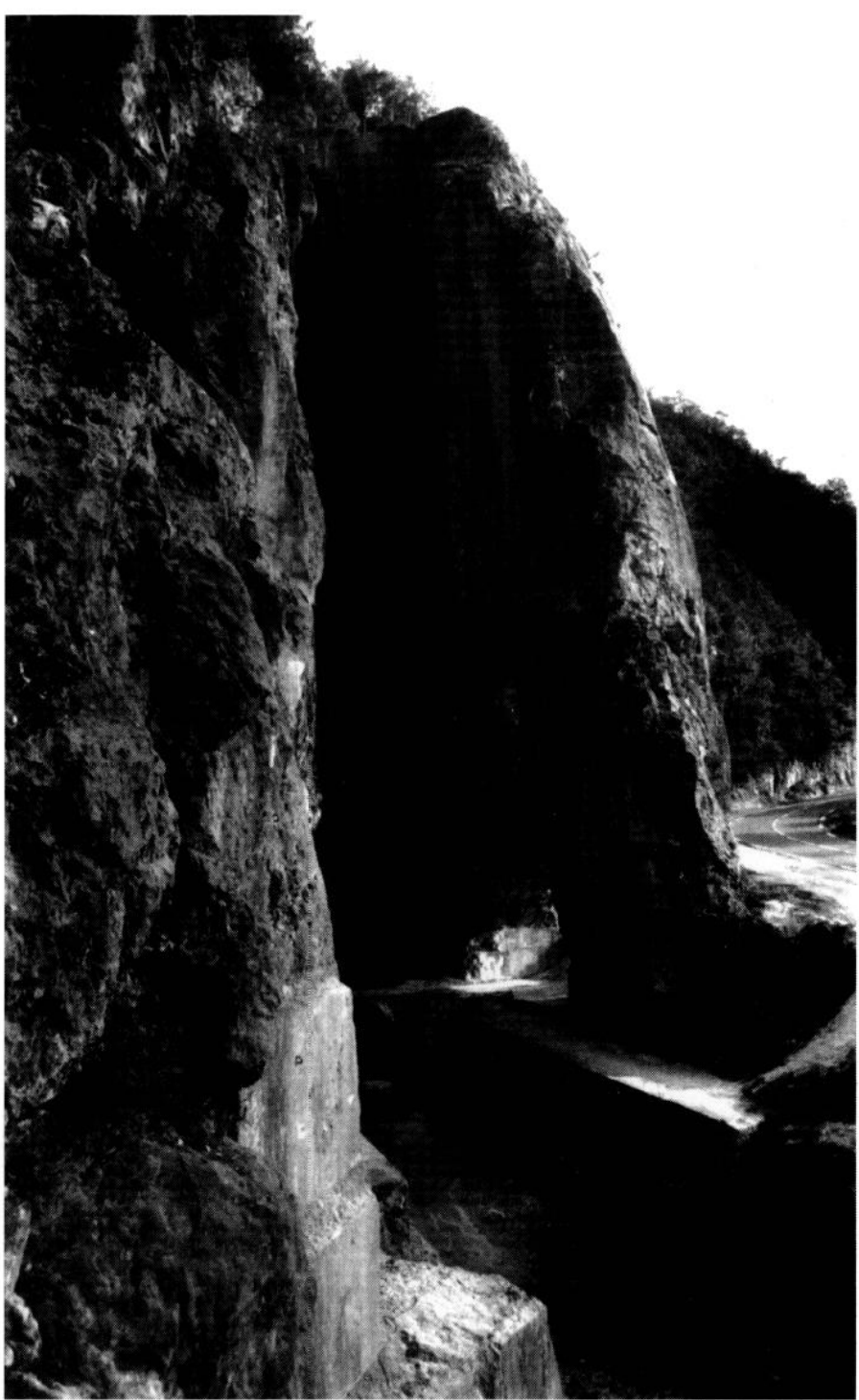

Fig. 47 - Carrière de La Pène-Saint-Martin à Saint-Béat, Haute-Garonne (photo J.-M. Fabre, CNRS).

établissements ruraux de la région de Réalville, au nórd de Montauban ${ }^{45}$. Les tableaux et graphiques sectoriels présentent la provenance des différents matériaux utilisés sur les sites archéologiques (fig. 48).

On peut remarquer d'emblée des proportions identiques pour les deux sites pyrénéens : à Valentine, comme à Saint-Bertrand-de-Comminges, les marbres blancs et gris représentent 86 à $88 \%$ des fragments inventoriés, les griottes $8 \%$ et les brèches du type de La Pène-SaintMartin 4,5\%, soit 12 ou $13 \%$ de marbres colorés. Étant donné l'importance des lots de placages étudiés, près de 1300 fragments, on peut considérer ces chiffres comme

45. Prospection de V. Cousi, en 1998. 

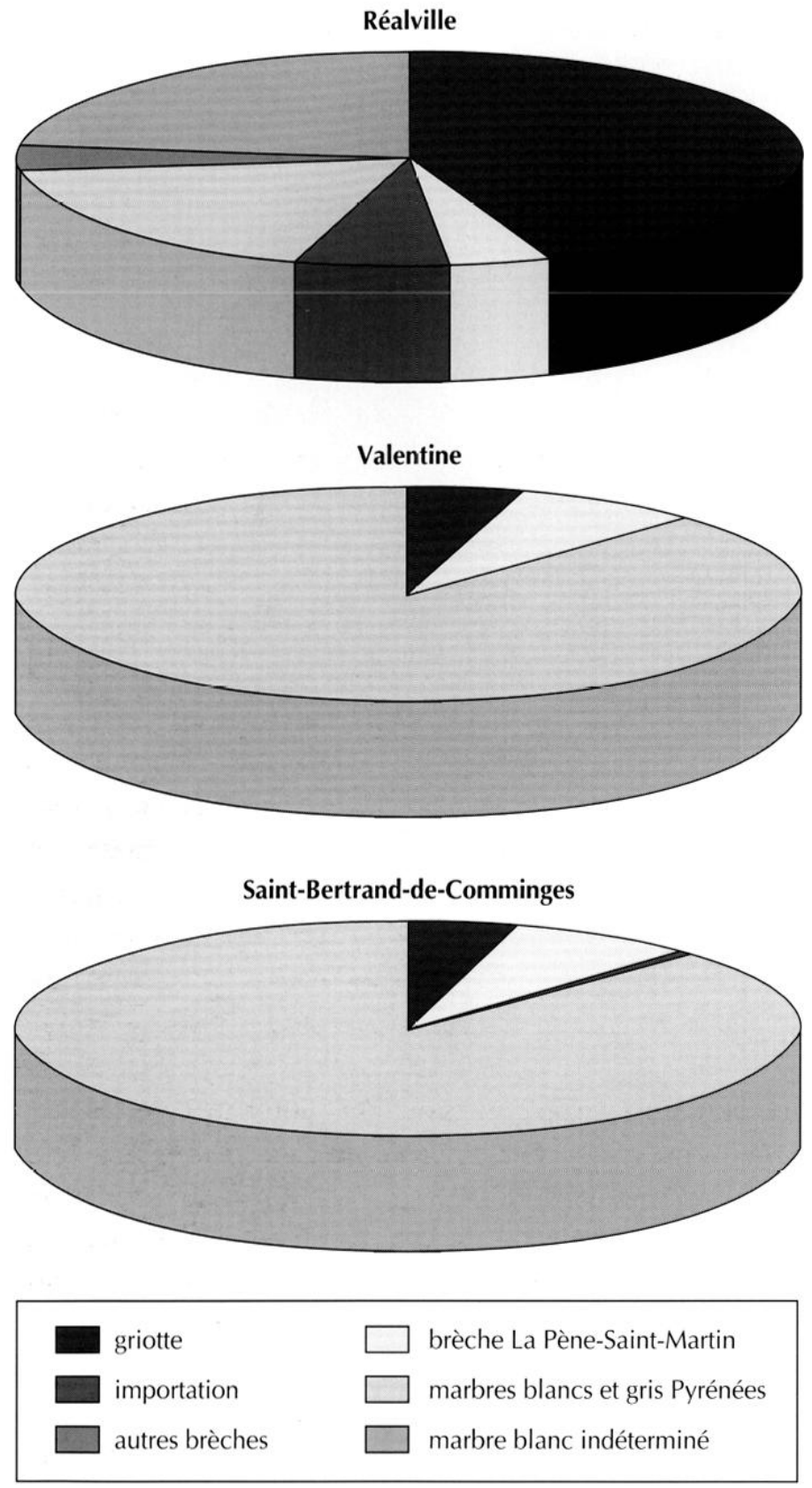

Fig. 48 - Placages de martres de la région de Réalville (Tarn-et(aronne), de Valentine et de Saint-Bertrand-de-Comminges (Haute(Garonne) (dessin J.-M. Fabre, (NRS).

représentatifs de l'utilisation des marbres pyrénéens dans la région de production.

Dans le secteur de Réalville, distant d'environ $200 \mathrm{~km}$ des carrières, les marbres pyrénéens sont toujours largement utilisés: ils représentent un minimum de $67 \%$, auquel il faut probablement rajouter une partie des $23 \%$ de marbres blancs de provenance indéterminée. On peut remarquer cependant l'importance relative des importations $(10 \%)$, pour des sites ruraux et une agglomération secondaire relativement modestes en comparaison des grands sites pyrénéens beaucoup plus luxueux.

Dans les deux régions, le rapport entre marbres blancs et gris et marbres colorés est totalement différent. À Réalville, les marbres de couleur représentent $58 \%$ des placages, contre 12 à $13 \%$ sur les sites proches des carrières. De même on peut constater que les griottes sont beaucoup plus utilisées (44\%) que les brèches $(4,3 \%)$.

Ces premières données permettent de faire quelques observations sur le marché régional de la pierre d'ornementation.

- Près des carrières, les architectes et les constructeurs utilisent abondamment le marbre local, notamment les marbres blancs et gris qui servent aux revêtements muraux et au dallage des sols de la plupart des pièces d'apparat. Les marbres étrangers sont pratiquement exclus du marché et les rares plaquettes importées devaient servir à composer de petits motifs décoratifs.

- À $200 \mathrm{~km}$ des carrières, le marbre est réservé à l'ornementation de certaines petites pièces luxueuses, dans les thermes par exemple. Dans ce cadre, les marbres colorés prennent plus d'importance, et, parmi eux, c'est la griotte qui semble la plus prisée. Il est probable qu'avec son esthétique bien particulière, ce marbre était le matériau qui rappelait le mieux les Pyrénées ainsi que la richesse du propriétaire des lieux.

\section{ÉTUDE TYPOLOGIQUE DU MOBILIER DE MARBRE}

Comme sur d'autres grandes villae de la région, telles que Chiragan (Martres-Tolosane) ou Montmaurin, les fouilles de Valentine ont exhumé un grand nombre de vestiges en marbre conservés dans un dépôt municipal. Ces collections, parfois mal inventoriées et pour lesquelles le contexte stratigraphique est bien souvent méconnu, offrent cependant d'importantes possibilités pour l'étude typologique des différents éléments de marbre en vue de la caractérisation des ateliers pyrénéens. À Valentine, les collections sont essentiellement composées de fragments de décors architecturaux, tels que moulures de corniches, chambranles, bases de colonnes et chapiteaux. Une étude minutieuse de cet ensemble a été entreprise : chaque objet a été 
décrit, mesuré, photographié, et son profil dessiné afin de constituer une banque de données informatisée. Cette base devrait permettre de confronter différents aspects morphologiques et techniques, mais les comparaisons sont encore difficiles, car le nombre de références est limité, et les publications utilisables sont relativement rares. Pour le sud-ouest de la Gaule, les travaux de R. Monturet, sur les marbres de Plassac et Séviac (commune de Montréal), constituent cependant des références précises et utilisables (Bost, Monturet, 1985 ; Monturet, Rivière, 1987, p. 193-217).

Aux côtés de séries classiques, telles que celle des chambranles architravés ou ioniques, d'autres groupes semblent moins courants (fig. 49, $\mathrm{n}^{\circ}$ 9883), mais il est cependant difficile de bien définir leur originalité, et donc prématuré de leur accorder un caractère pyrénéen. L'étude en cours sur d'autres sites régionaux, notamment à Montmaurin, devrait permettre de mieux distinguer les pratiques des tailleurs de marbre locaux.

Très répandus dans la région, les mortiers et vasques de marbre ne semblent pas avoir fait l'objet d'une production standardisée (fig. 49). La variété des formes observées ferait plutôt penser à une valorisation des rebuts de matériaux de construction sur les chantiers, ou des rebuts d'exploitation dans les ateliers près des carrières.

Sur plus de 40 modénatures complètes, deux grands types de moulures de corniche ont été distingués. Le plus simple se compose d'une seule moulure curviligne, doucine ou cavet, encadrée par des bandeaux, avec parfois un listel plat ou déversé, tandis que le second type, de dimensions plus importantes, comprend deux moulures curvilignes. Le nombre de variantes relativement élevé rend caduque toute classification précise et met en valeur la diversité des décors utilisés sur un même site. D'après l'étude en cours sur le mobilier de la villa de Montmaurin, où plusieurs des éléments retrouvés en fouille étaient en cours de taille, les moulures de couronnement étaient fabriquées sur le chantier de construction, peut-être par un atelier itinérant. Le matériau était apporté de la carrière sous forme de barres découpées à la scie, dont la section correspond au gabarit des moulures.

Environ 20 fragments de pilastres sont conservés à Valentine. Tous présentent un entraxe de $45-50 \mathrm{~mm}$ et la seule forme complète présente six rudentures (fig. 50, $\mathrm{n}^{\circ}$ 9894). Sur une plaque de marbre, une base de pilastre est représentée en bas-relief ; elle est ornée de deux caissons moulurés, décor caractéristique des ateliers pyrénéens (Braemer, 1969, p. 100).

L'étude des éléments de colonnades, trop peu nombreux, n'a pour l'instant donné aucun résultat (Fabre, Lucas, 2001).

Sur certains vestiges, notamment les pilastres, les tailleurs ont utilisé le pied romain comme unité de mesure, mais il est difficile de mener une étude de métrologie sur cette collection. Bien souvent, les fragments conservés sont de taille réduite, et la marge d'erreur, liée à la qualité du travail du marbrier et à l'état de conservation du vestige, semble importante. Seul un travail portant sur les gabarits de chaque série d'objets semble envisageable.

Sur l'ensemble de la collection de Valentine, la qualité du travail des marbriers (régularité dans les modénatures, traitement des surfaces...) apparaît très variable. Ainsi, par exemple, certaines moulures sont finement polies tandis que d'autres n'ont pas atteint ce stade de finition et présentent de nombreuses traces d'outils, comme la gradine, le ciseau grain d'orge ou le ciseau. Ces différences ne semblent pas liées à la nature ou à la fonction des objets, mais plutôt à une pratique particulière sur le chantier, due peut-être à des délais de mise en ouvre trop courts ou à des coûts trop élevés. Les nombreuses imperfections constatées dans le travail de la pierre viennent contrebalancer l'abondance et la richesse des marbres sur le site. Le commanditaire a utilisé une grande quantité de matériaux provenant des gisements voisins, mais, pour des raisons techniques ou économiques, il n'a pu assurer un façonnage régulier et de qualité. Plus globalement, cet aspect montre bien la prépondérance de deux éléments dans le commerce et l'utilisation du marbre: le transport des matériaux, d'une part, et le façonnage et la mise en ceuvre sur le chantier de construction, d'autre part.

\section{L'IMPACT DE L'EXPLOITATION ANTIQUE DU MARBRE SUR LA VIE DES VALLÉES}

\section{UN DÉVELOPPEMENT PRÉCOCE ET CONTINU}

L'archéologie, comme l'épigraphie et les textes, porte d'abondants et éloquents témoignages de la réalité et du volume de l'activité marbrière dans les vallées centrales 


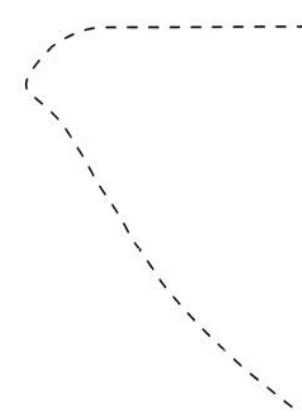

98141
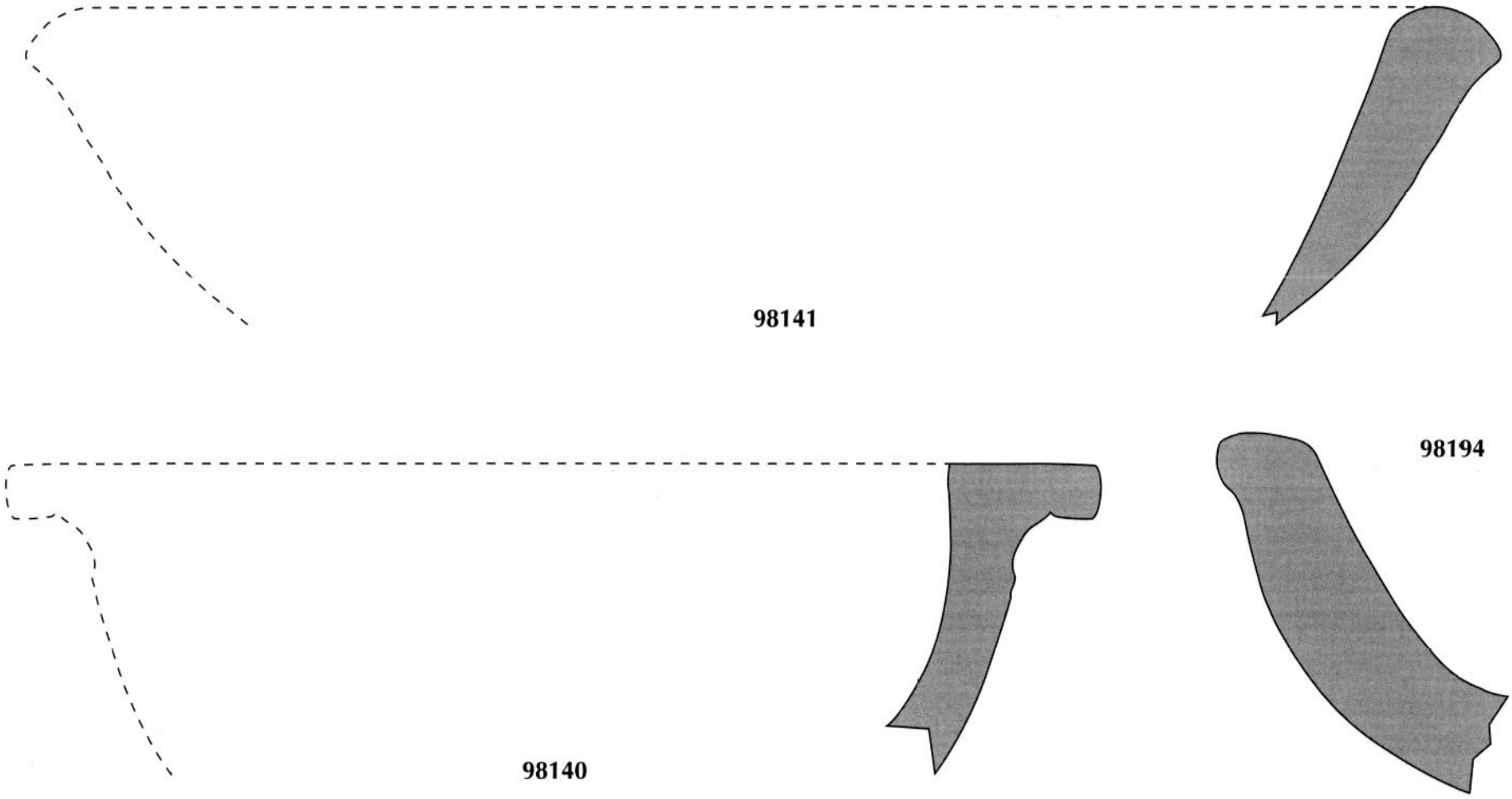

98140
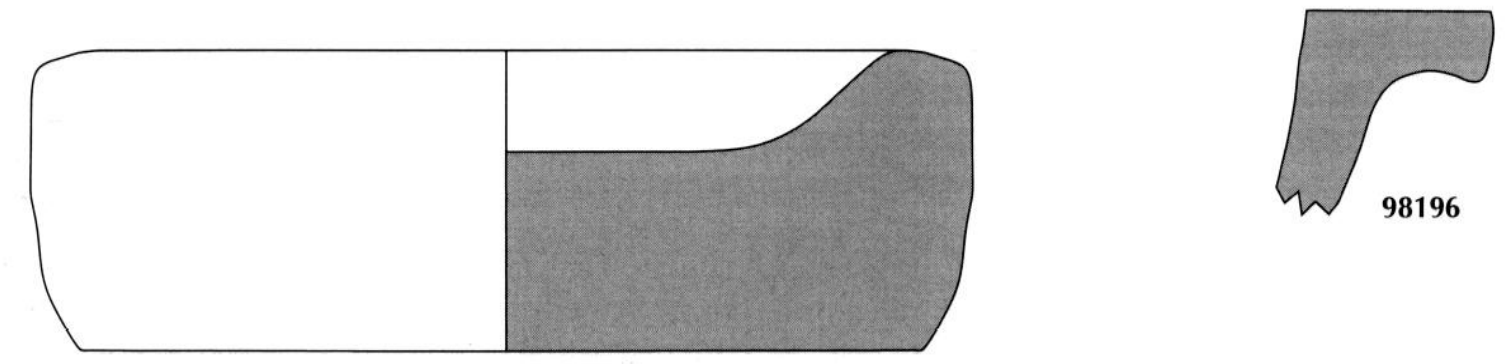

98146

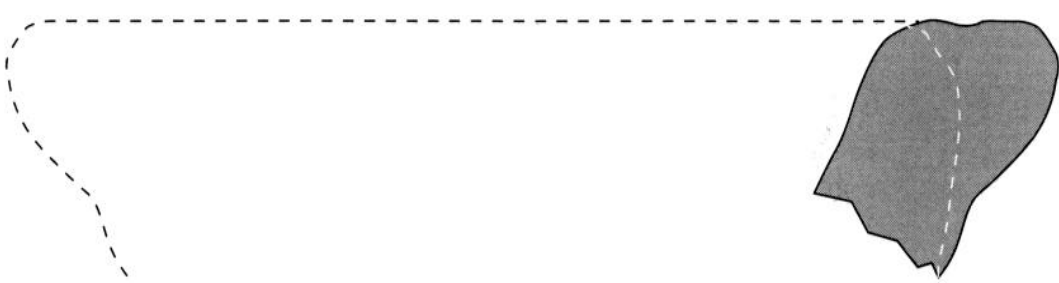

98266

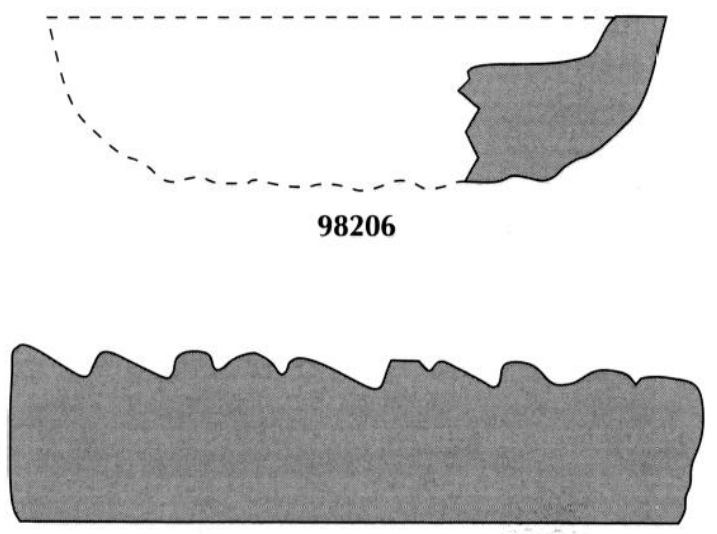

98112

$5 \mathrm{~cm}$

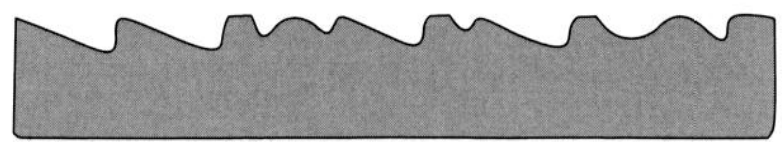

9883

98116

Fig. 49 - Valentine, Haute-Garonne : vasques, mortiers et chambranles de marbre (dessin J.-M. Fabre, CNRS). 
Fig. 50 - Valentine, Haute-Garonne : fragments de pilastres (dessin J.-M. Fabre, CNRS).

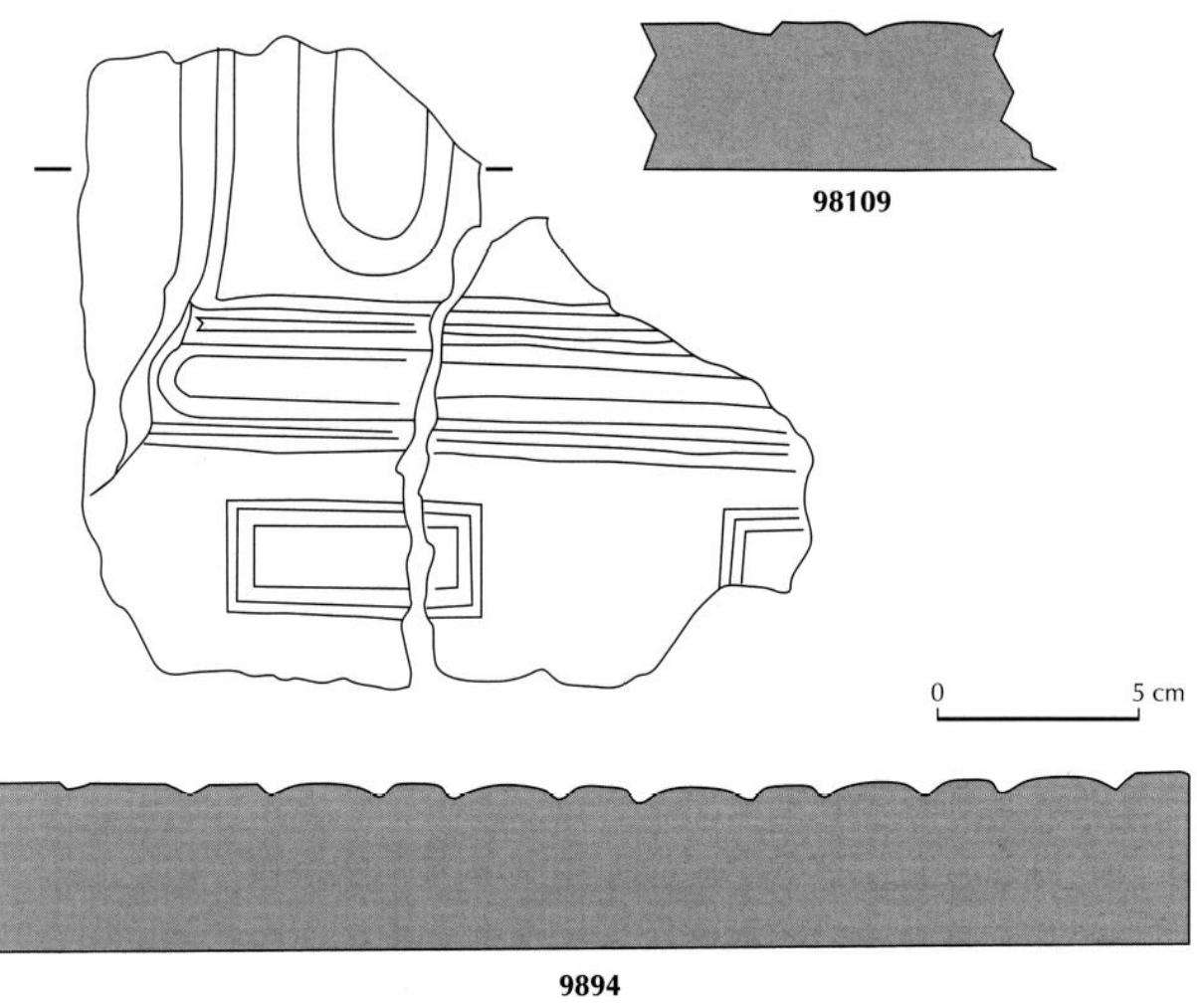

des Pyrénées. Plusieurs découvertes ou recherches récentes offrent en la matière des jalons chronologiques qu'il convient de rappeler.

Un premier rapprochement est celui de l'hypothèse, proposée par E. Boube, sur la chronologie et le sens du célèbre trophée de Saint-Bertrand-de-Comminges (Boube, 1996) avec les analyses, effectuées par C. Costedoat (1992), du marbre de ces pièces. ¿̀ l'initiative de J.-L. Schenck, conservateur du musée archéologique départemental de Saint-Bertrand-deComminges, le groupe statuaire a fait l'objet, depuis 1998, d'une remarquable restauration et d'une exposition originale, qui permet de revoir, dans un état rénové et une présentation renouvelée, ces pièces exceptionnelles. L'analyse historique développée par E. Boube, si elle ne bouleverse pas les données antérieures établies par G.-C. Picard, n'en modifie pas moins sensiblement le contenu. S'appuyant sur des critères archéologiques empruntés aux fouilles récentes du temple du forum à Saint-Bertrand (Badie et al., 1994), sur une nouvelle hypothèse de restitution et sur une analyse stylistique des pièces, E. Boube propose de voir dans le monument triomphal une œuvre commandée par le pouvoir impérial au moment de la réorganisation des Gaules par Auguste et Agrippa, après 16-13 avant notre ère (Boube, 1996, p. 40-44). Cette analyse est corroborée par les données archéologiques et historiques récentes, qui témoignent du changement, à cette période, de statut de la ville, promue capitale de cité, et du développement concomitant d'un urbanisme d'envergure (Sablayrolles, 1997, 1999, à paraître ; Sablayrolles, Tassaux, 1999). Les travaux de C. Costedoat, réalisés dans le cadre de sa thèse (Costedoat, 1992, p. 100-103), ont mis en évidence l'origine du marbre des pièces du trophée : les données croisées de l'analyse isotopique et de la cathodoluminescence ainsi que le test discriminant évoqué supra désignent formellement la carrière du Cap del Mount, située sur la rive droite de la Garonne, à Saint-Béat (fig. 45, $\mathrm{n}^{\circ} 15$ ), et non pas des carrières de la Montagne d'Arri, sur la rive gauche, comme on le pensait jusqu'ici (Braemer, 1969, p. 81-82 ; Bedon, 1984, p. 36). Il faut en conclure que le pouvoir impérial, quand il décida d'ériger dans la capitale des Convènes un groupe statuaire de prestige, destinè à marquer de son empreinte l'urbanisme naissant, utilisa le marbre blanc local. Cela revient à dire que, si elles n'étaient pas déjà en fonction, hypothèse qu'on ne saurait écarter, les carrières de marbre de Saint-Béat furent ouvertes au plus tard dès la deuxième décennie avant notre ère ${ }^{46}$.

46. On ne peut s'en tenir aux affirmations trop catégoriques de R. Bedon, pour qui l'ouverture des carrières de Saint-Béat était conco- 
Fig. 51 - «Sarcophage à l'orante ", église Saint-Just à Valcabrère, Haute-Garonne (photo D. Martin).

Fig. 52 - Sarcophage historié, église Saint-Just à Valcabrère, Haute-Garonne (photo D. Martin).

Un jalon récemment découvert témoigne de la permanence de l'activité marbrière durant l'Antiquité

mitante de l'érection du trophée (Bedon, 1984, p. 36 et 64). La haute vallée de la Garonne était en effet sous contrôle romain depuis la réorganisation de la Gaule Transalpine par Pompée, après les guerres contre Sertorius. Si elle n'avait encore ni le statut ni l'apparat d'une capitale, la bourgade de Lugdunum, dont la création, en 72 avant notre ère, est attribuée à Pompéc par le texte tardif du Père de l'Église Jérôme (Contre Vigilance, éd. Migne, t. XXIII, col. 353), n'en tardive et de l'existence d'ateliers locaux produisant des pièces de valeur (Schenck, 1991 ; Dieulafait, Guyon,

constituait pas moins une garnison frontière, tout à fait susceptible d'avoir exploré les ressources naturelles des vallées avoisinantes et d'avoir mis en chantier, pour des besoins locaux, épigraphiques notamment, une exploitation marbrière. Plus d'un demi-siècle sépare la modeste installation pompéienne de sa transformation en capitale par Auguste, laps de temps suffisant pour le développement de l'activité extractive. 


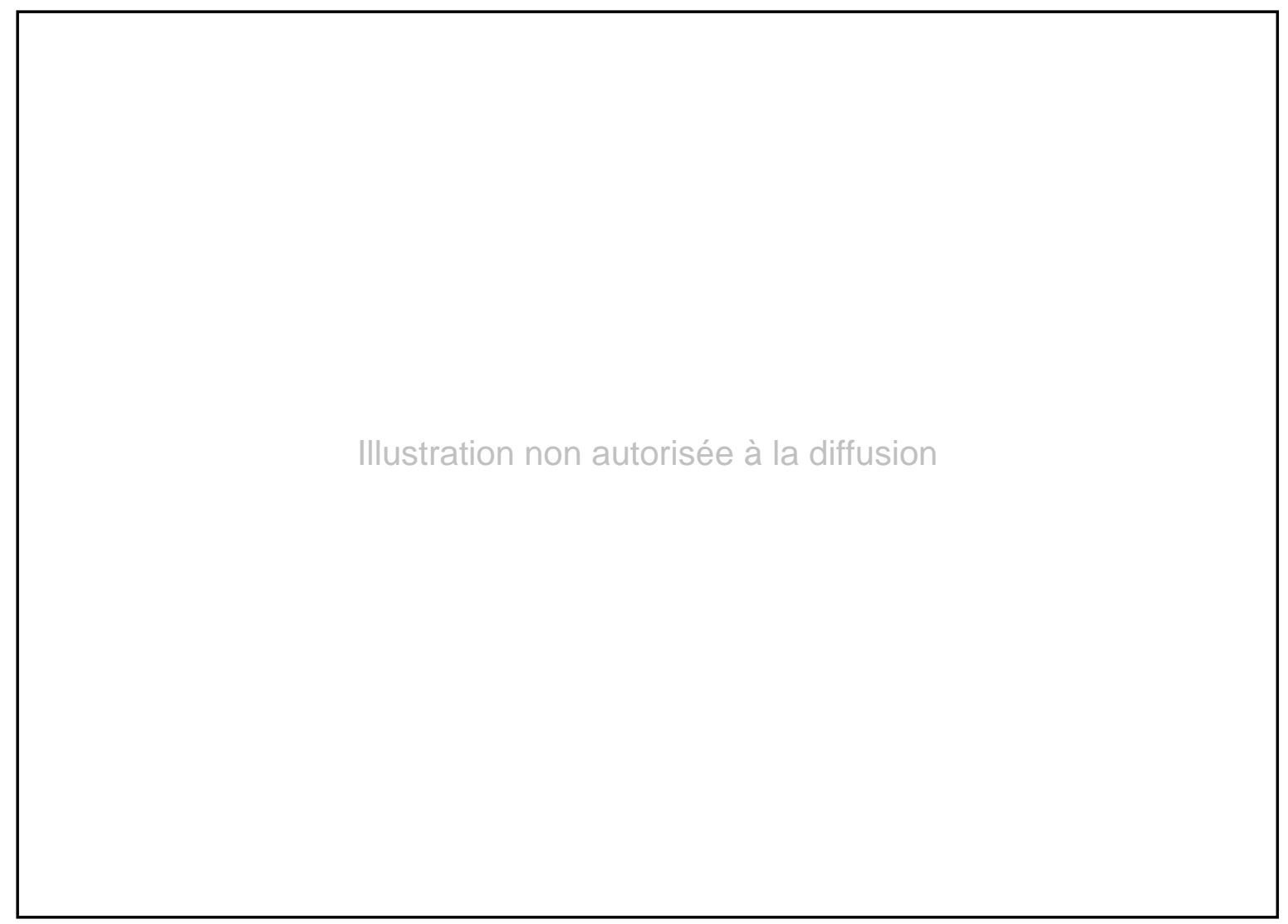

Fig. 53 - Sarcophage ébauché de la genèse, église Saint-Just à Valcabrère, Haute-Garonne (photo D. Martin).

1991). Lors d'une fouille de sauvetage, entreprise avant des travaux de drainage le long du mur gouttereau septentrional de l'église Saint-Just à Valcabrère, furent découverts trois fragments de sarcophages historiés en marbre. Le premier, le «sarcophage à l'orante » (fig. 51), est un sarcophage où se reconnaissent des thèmes chrétiens (miracles de la multiplication des pains et de la guérison du paralytique, arrestation de saint Pierre). Le deuxième (fig. 52) est un sarcophage de sens mystérieux, où se combinent thèmes funéraires paiens traditionnels (le lit et le banquet funéraires, le volumen, symbole de culture) et thèmes potentiellement chrétiens (orante, lion qui pourrait être celui de Daniel). Le troisième (fig. 53) est simplement ébauché et l'on y reconnaît une scène classique de la genèse, avec Adam et Ève de part et d'autre de l'arbre au serpent, et l'ébauche d'un lion, qui présente bien des similitudes avec la représentation du sarcophage précédent. Le "sarcophage à l'orante ", par la qualité de la pièce et le soin de l'exécution, est rapproché par C. Dieulafait et J. Guyon des œuvres romaines ou arlésiennes (Dieulafait, Guyon, 1991, p. 58). Mais, comme le reconnaissent les auteurs, le marbre, analysé par C. Costedoat, vient bien des carrières de
Saint-Béat (Costedoat, 1992, p. 104-105). Les deux autres sarcophages, d'une facture plus originale mais aussi moins parfaite dans l'exécution, appartiennent à un même atelier, voire à une même main (Dieulafait, Guyon, 1991, p. 68). Or, l'ébauche abandonnée du sarcophage d'Adam et Ève prouve bien l'existence d'un atelier local, auquel il faut rattacher également, par conséquent, le fragment de cuve du deuxième sarcophage. Ces découvertes exceptionnelles, datées de la charnière des III $^{\mathrm{C}}$ et IV s. (Dieulafait, Guyon, 1991, p. 59 et 65), témoignent donc bien de la pérennité, à cette époque, de l'extraction du marbre dans les carrières de Saint-Béat et de l'existence, à proximité des nécropoles de la capitale Lugdunum, sur lesquelles est édifiée l'église Saint-Just, d'un atelier de qualité, qui exécutait des sarcophages à panneaux historiés.

La chronologie de la basilique paléochrétienne, renouvelée par les fouilles récentes (Guyon, Paillet, 1991), offre un dernier jalon, qui repousse au-delà de l'Antiquité tardive le terminus de l'exploitation marbrière de Saint-Béat. Les stratigraphies de l'édifice ont en effet démontré qu'un premier édifice à chevet carré, construit dans la première moitié du $V^{c}$ s., avait été agrandi par un 
narthex et un chevet polygonal, peut-être lorsque la basilique devint espace funéraire. À cette deuxième phase appartiennent les nombreux sarcophages en marbre de Saint-Béat enfouis sous le sol de la seconde basilique. Un terminus post quem à cette pratique, et donc à l'usage du marbre de Saint-Béat pour la fabrication de sarcophages, a été donné par la datation au ${ }^{14} \mathrm{C}$ d'une de ces sépultures : le VII ${ }^{\mathrm{c}} \mathrm{s}$. de notre ère. L'écart s'est donc resserré entre l'exploitation antique des carrières et l'exploitation médiévale, attestée dès la période préromane comme l'ont montré les travaux de J. Cabanot et C. Costedoat sur les chapiteaux (Cabanot, 1995).

S'il est impossible, en l'état actuel des données, d'évaluer le rythme de la production (continue ou discontinue) pour les phases tardives, si des périodes d'interruption ne sont pas à exclure entre les derniers des jalons à notre disposition, en revanche, pour les trois premiers siècles de notre ère, le marché proche constitué par Lugdunum fut en constant essor, comme le révèlent les fouilles récentes. À la phase initiale d'édification de la capitale, qui, au gré des impulsions du pouvoir impérial, de l'adhésion au modèle romain des aristocraties locales et de leurs disponibilités financières, dura au moins un demi-siècle, succédèrent des chantiers de rénovation des bâtiments anciens ou de construction de nouveaux édifices. Le plus ancien monument en marbre connu à l'heure actuelle est, comme il vient d'être rappelé, le trophée, et son érection, peut-être à proximité d'un autel du culte impérial (Badie et al., 1994, p. 29-32 et 113-114), constitua une sorte de préalable à l'urbanisation. Le temple, construit dans le premier quart du $\mathrm{I}^{\mathrm{er}} \mathrm{s}$. de notre ère, était en calcaire, mais plusieurs fragments de plinthes, de placages et de moulures, découverts parmi les débris du chantier, montrent que les murs du portique au moins possédaient déjà ce type de revêtement (Badie et al., 1994, p. 47). Les thermes du forum, dont la première construction était antérieure à celle du temple, ne connurent pas moins de trois états successifs dans le cours du $\mathrm{I}^{\mathrm{cr}} \mathrm{s}$. et la décoration architecturale en marbre y était abondante (Aupert, Monturet, 2001). Le théâtre, durant la même première moitié du $I^{\text {er }} \mathbf{s}$., fut l'objet d'une réfection après sa construction : dans l'un et l'autre état, les gradins étaient revêtus de marbre de Saint-Béat (Janon, 1997). Un édifice public de la périphérie urbaine, schola ou palestre édifiée au début du $\mathrm{I}^{\mathrm{er}}$ s., possédait également une riche décoration architecturale en marbre: l'immense bassin mosaiqué
(17,80 m x 10,20 m), qui occupait le centre de la cour, était entièrement revêtu de marbre ${ }^{47}$. On pourrait multiplier les exemples avec les thermes du Nord (fin du $\mathrm{I}^{\mathrm{er}}$ s.), la grande domus qui remplaça, au milieu du $\mathrm{II}^{\mathrm{c}} \mathrm{s}$., l'édifice de Coupéré, le camp militaire du début du III ${ }^{\mathrm{e}} \mathbf{s}$. Le développement continu de l'urbanisme dans la ville antique, qu'il ne faut plus imaginer sous forme de phases d'activité suivies par de longues périodes d'immobilisme, mais bien comme un processus permanent témoignant de la vitalité économique de la cité, constitua pour les carrières de marbre une demande ininterrompue. Les études des collections de Montmaurin et Valentine, la chronologie de la villa de Séviac à Montréal, où l'usage du marbre pyrénéen est largement attesté (Monturet, Rivière, 1987), montrent que cette demande ne s'interrompit pas durant le IV $\mathbf{s}$.

$\mathrm{Si}$ la production diffusée régionalement permet d'induire une production permanente durant les quatre premiers siècles de notre ère au moins pour les carrières de Saint-Béat, et une activité au moins sporadique pour les deux ou trois siècles suivants, il reste peu de traces de la commercialisation. L'existence de deux voies, sur les rives droite et gauche de la Garonne, laisse ouverte la possibilité d'un transport terrestre pour les pièces les moins volumineuses. Le transport fluvial, possible sous forme de radeaux et probable pour les blocs monumentaux, n'a pu être illustré archéologiquement, malgré des prospections minutieuses des berges de la Garonne aux abords des carrières, et la seule attestation épigraphique éventuelle de cette radellerie (Sablayrolles, Schenck, 1988, $\mathrm{n}^{\circ} 48$ ) reste sujette à caution, plusieurs interprétations pouvant être données de l'inscription (ibidem ; Fabre, Sablayrolles, 1995, p. 151). Si les traces concrètes de la circulation des productions font défaut, l'impact de cette économie sur la vie des vallées n'en est pas moins sensible.

\section{LA MARQUE DE LA PIERRE DANS LE BASSIN DE LA GARONNE}

Le mobilier épigraphique du sanctuaire de la carrière de Rapp, fouillée en 1946 par B. Sapène et récemment réétudiée (Sapène, 1946 ; Fabre, Sablayrolles, 1995), met en évidence une participation de la population indigène

47. Données issues des fouilles 2000 du site de Coupéré (SaintBertrand-de-Comminges), dirigées par R. Sablayrolles. 
à l'exploitation, mais une acculturation plus développée, chez cette population, à la langue et à l'onomastique latines. Ce métissage plus marqué des cultures s'explique probablement par la présence, au moins ponctuelle, de main-d'ceuvre ou d'encadrement romains et par l'intensité des échanges qui mettaient en contact permanent le monde de la carrière et celui de la ville, pôle par excellence de la diffusion de la romanité. Sans reprendre ici le détail de l'étude (Fabre, Sablayrolles, 1995, p. 151-156), rappelons que plusieurs inscriptions attestent le lien entre les marbriers et le sanctuaire (dédicaces par des marmorarii, en groupe ou isolés, et par des officinatores, évocation d'activités techniques de l'extraction ou de la taille). Ces fidèles honoraient un dieu local, Erriape, probable génie préromain de la montagne, transformé en protecteur des marbriers et de l'exploitation, et rapproché, par le fait, de dieux romains comme Hercules Saxanus et Silvain. L'onomastique de ce groupe de fidèles révèle une latinisation supérieure à la moyenne de la population locale, malgré la persistance de quelques noms aquitains, normalement majoritaires dans les dédicaces aux petites divinités indigènes. Ce processus, très marqué dans le corpus d'inscriptions du sanctuaire de la carrière, s'observe également, mais atténué, dans deux pratiques typiques des vallées des Pyrénées centrales, et surtout du bassin de la Garonne : les autels votifs et les auges cinéraires.

Il n'existe pas, pour l'heure, d'inventaire exhaustif des autels votifs de l'Aquitaine méridionale: seul, l'ensemble du musée archéologique départemental de Saint-Bertrand-de-Comminges a fait l'objet de publications récentes (Sablayrolles, Schenck, 1988; Autels votifs, 1991). Le recensement, même approximatif, de ces vestiges est rendu difficile par leur dispersion. Les musées régionaux (Saint-Bertrand-de-Comminges, Toulouse, Tarbes, Bagnères-de-Bigorre, Bagnèresde-Luchon) en détiennent près de 550 , dont quelque 350 portent une inscription. Une évaluation des éléments dispersés dans les réemplois in situ et les collections particulières laisse penser qu'il faut chiffrer à 800 au moins, et peut-être à 1000 l'ensemble du corpus. À plus de $80 \%$, ces autels ont été découverts dans les vallées pyrénéennes ou dans le piémont immédiat. Leur diffusion couvre bien sûr la capitale, Lugdunum, et les sanctuaires des vici ou pagi du piémont, mais également les sanctuaires d'altitude, comme celui du Mont Sacon, à $1500 \mathrm{~m}$, ou celui de la divinité Ageio, entre Neste et

\section{Illustration non autorisée à la diffusion}

Fig. 54 - Autel votif dédiè au dieu Erriape, carrière de Rapp à Saint-Béat, Haute-Garonne (photo K. Schenck-I)avid).

Adour, à $1200 \mathrm{~m}$ (Schenck, à paraître). Ces objets rituels sont de tailles diverses, du bel autel monumental, dont la hauteur peut atteindre $0,80 \mathrm{~m}$ à $1 \mathrm{~m}$, et dont le décor comme le texte sont soignés (autel à Erriape) (fig. 54), au modeste ex-voto de $0,10 \mathrm{~m}$ à $0,20 \mathrm{~m}$ de haut, souvent anépigraphe et de forme grossière, à peine ébauchée (autel de Tibiran-Jaunac) (fig. 55). Ces dernières productions laissent envisager l'utilisation, pour leur ćlaboration, de déchets de carrière, que les marbriers récupéraient et façonnaient peut-ĉtre à destination de la population locale. L'usage de ce type d'offrande dans les sanctuaires témoigne, par le recours à la symbolique du sacrifice, à l'écriture latine et aux canons du décor architectural du monde méditerranéen, de l'adoption de symboles religieux romains, et donc, peut-être, d'une mutation du rituel. Cette mutation s'observe aussi dans la personnalité des divinités qui subissent, par le processus 


\section{Illustration non autorisée à la diffusion}

Fig. 55 - Autel volif découvert à Tibiran-Jaunac, Hautes-Pyrénées (photo K. Schenck-I)avid).

classique de la contaminatio, l'influence de divinités romaines, comme dans le cas d'Erriape avec Silvain et Hercules Saxanus, qui a été évoqué. Le contraste dans la répartition de ces témoins lapidaires est frappant: le haut bassin de la Garonne, en amont de la capitale Lugdunum, compte plus de 200 exemplaires, alors que celui de la Neste, à l'ouest, en amont de Saint-Laurent, n'en compte déjà plus que 9, et celui de l'Adour à peine 5 , auxquels il faut ajouter, il est vrai, les quelque 25 pièces découvertes par J.-I. Schenck au sanctuaire des Arès, dans les hautes Baronnies (Schenck, à paraître). Vers l'est, l'ensemble du bassin du Salat n'a livré que cinq pièces et le phénomène paraît absent des Pyrénées orientales et occidentales. La cartographie est donc éloquente et cette surreprésentation du bassin de la Garonne (auquel on pourrait ajouter la centaine d'autels votifs de
Saint-Bertrand-de-Comminges et Valcabrère ainsi que les nombreux autels du proche piémont) illustre l'ampleur du phénomène. La première explication est naturellement la facilité d'accès au matériau : les riverains de la Garonne pouvaient se procurer à meilleur prix des supports en marbre de Saint-Béat pour leurs dévotions. Cela explique la diffusion de ces objets dans des espaces a priori inattendus, comme les sanctuaires d'altitude, et dans des couches assez diverses de la société : si plusieurs autels sont manifestement des dépôts de familles aristocratiques locales, qui jouaient un rôle non négligeable dans la diffusion de la culture latine, religieuse en particulier (Fabre, 2000b), d'autres, plus modestes, ont été dédiés par les utilisateurs de la montagne, forestiers (Sablayrolles, 1993), métallurgistes (Beyrie et al., 2000) ou bergers (Fabre, 1993). I'impact économique de la pierre, à la fois directement, par la facilité d'accès au matériau pour les populations locales, et indirectement, par les échanges intenses qu'il engendrait dans l'axe des vallées, eut donc des répercussions sur les comportements religieux, dans le domaine du rituel, avec la multiplication des autels votifs, mais aussi sans doute, grâce à ce canal, dans des domaines plus profonds comme ceux de la personnalité des dieux et de la culture latine des fidèles.

Ce sont des observations similaires que suggère l'étude de ces monuments si typiques du paysage pyrénéen antique que constituent les auges cinéraires, réceptacles en marbre des urnes ou vases qui contenaient les cendres des défunts. L'analyse récente qu'en a proposée A. Laurens fournit désormais un inventaire exhaustif du corpus et offre des perspectives novatrices de réflexion (Laurens, 1998, 1999, 2000). Le catalogue établi par A. Laurens recense 399 auges cinéraires et plaques d'obturation de coffres maçonnés, et la carte de répartition qu'elle propose est particulièrement éloquente (Laurens, 1998, carte 2). En effet, parmi ces pièces, 291 (73\%) appartiennent au haut bassin de la Garonne, en amont de la capitale Iugdunum. La capitale elle-même et ses nécropoles (Valcabrère) en ont fourni 10 exemplaires. Ia vallée de la Garonne aux abords de Saint-Bertrand-de-Comminges, depuis Montoussé, à $10 \mathrm{~km}$ vers l'est, jusqu'à Martres-Tolosane, à $40 \mathrm{~km}$ à l'ouest, a livré 24 spécimens. Un groupe de 58 autres se répartit, de façon dispersée, dans un rayon de 20 à $40 \mathrm{~km}$ au nord de Saint-Bertrand, le long des vallées qui descendent du piémont : la Louge (12), 
la Save (36), la Gimone (4), l'Arrats (2), le Gers (2), la Baise (2), la cuve la plus éloignée des Pyrénées ayant été découverte à Castelnau-Barbarens, quelque $50 \mathrm{~km}$ vers le nord, dans la vallée de l'Arrats. Le bassin de la Neste, à l'est, n'a livré que 3 auges cinéraires et celui du Salat, à l'ouest, seulement 7 . Hors de la zone pyrénéenne, ce type de monument n'est attesté qu'à Toulouse ( 3 exemplaires certains et 3 hypothétiques). Il s'agit donc bien d'une production typique des carrières de la vallée de la Garonne, dont la diffusion, très importante localement, s'est ensuite limitée aux axes naturels des vallées, ne dépassant pas, à la seule exception de Toulouse, la quarantaine de kilomètres au-delà du piémont.

Ces auges présentent fréquemment des décors sur leur face antérieure, où se mêlent motifs géométriques ou végétaux stylisés, bustes raides et naifs de personnages souvent associés par deux ou trois (fig. 56) et, parfois, objets de la vie quotidienne ou du rituel religieux (Laurens, 2000). Ce décor est structuré dans des niches en demi-cercle ou dans un cadre architectural qui symbolise souvent une façade encadrée de deux colonnes ou pilastres. Comme l'a fort bien montré A. Laurens (1998, p. 593-604), et contrairement aux opinions antérieures, qui cherchaient dans d'hypothétiques traditions préromaines qu'aucun argument archéologique ne venait étayer l'origine de ces monuments funéraires (Hatt, 1942-1943), ceux-ci sont le fruit d'un métissage entre des pratiques liées au rituel funéraire romain et des traditions autochtones, peut-être religieuses, mais plus sûrement sociales. À l'univers romain de la mort appartient l'usage de la pierre, qui, seule, assurait un espoir d'éternité, dans une religion de la collectivité, formaliste et ritualiste, où la mort de l'individu n'était qu'épisode anecdotique. Le portrait dans la niche, le caractère architectural de la face antérieure rappellent, en miniature, les traditionnels tombeaux maçonnés de l'Italie et il est légitime de penser que les moins pauvres des montagnards achetaient dans ces auges cinéraires des succédanés des tombeaux ou mausolées qu'ils ne pouvaient s'offrir pour marquer leur position dans la petite collectivité qui était la leur. À la même veine latine, celle du banquet funéraire et des libations, pourraient appartenir les scènes nombreuses où les personnages tiennent dans leurs mains cruches et gobelets. L'originalité du geste et la fréquence de la représentation n'excluent cependant

\section{Illustration non autorisée à la diffusion}

Fig. 56 - Auge cinéraire à personnages, Saint-Pé-d'Ardet,
Haute-Garonne (photo A. Laurens).

pas un rituel local. D'une tradition proprement pyrénéenne relèvent la stylisation originale et la raideur quelque peu naïve des portraits, leur association dans un groupe familial qui paraît plus restreint que la familia latine, l'utilisation relativement fréquente d'objets de la vie quotidienne, parmi lesquels sont représentés, entre autres, les outils des métiers de la montagne : haches, doloires et scies de bûcherons, massettes et pics de carriers, marteaux et pinces de forgerons, armes de chasseurs (Laurens, 2000). On se gardera de toute interprétation sur les symboles végétaux, animaux ou géométriques et sur l'éventuelle signification symbolique de représentations animales (oiseaux) : l'absence de texte empêche de fonder toute hypothèse dans ce domaine et les tentatives d'interprétation relèvent, dès lors, plus de l'imagination ou des fantasmes du chercheur que d'une analyse rigoureuse des sources.

Contentons-nous des certitudes, pour frustrantes qu'elles soient aux yeux des amateurs de mysticisme: l'artisanat du marbre a engendré, autour des carrières, l'usage de monuments funéraires originaux dans une population modeste, dont les moins pauvres pouvaient économiquement accéder à cette forme de richesse ostentatoire post mortem. Le regroupement fréquent de 
ces vestiges dans des nécropoles de soulane les rattache majoritairement aux vici ou pagi des vallées ${ }^{48}$, dont la vie s'était transformée dans l'univers d'échanges que constituait l'Empire romain, mais dont les traditions restaient plus vivaces que dans la ville ou dans les plaines, où la culture dominante s'était plus largement imposée. Expression de cette transformation, l'auge cinéraire en marbre, qui alliait traits romains et traditions pyrénéennes et permettait, dans les petites agglomérations montagnardes, la manifestation d'une différenciation sociale, fut en même temps vecteur et instrument de cette transformation. La carrière et l'atelier, présence physique des commandes de la ville dans les vallées montagnardes, furent la vitrine des modes de représentation urbains pour des populations locales, directement ou indirectement associées à ces activités marbrières. Avec le mode de représentation, qu'il soit de nature sociale ou religieuse, se répandit aussi la culture qui l'avait créé. Élément essentiel des mutations économiques de la vallée de la Garonne dans l'Antiquité, l'exploitation des carrières de marbre fut donc également, comme d'autres secteurs de l'économie montagnarde, un vecteur actif d'acculturation.

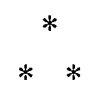

Ce tour d'horizon des travaux récents sur l'exploitation antique du marbre dans les Pyrénées ne se veut pas, nous l'avons dit, une synthèse globale et définitive, encore impossible à réaliser, mais un état de la question, qui mette en lumière les acquis et les lacunes, les potentialités et les impasses.

La prospection de terrain a atteint ses limites sur les vallées des Pyrénées centrales, dans les bassins du Salat, de la Garonne et de l'Adour. La cartographie actuelle, commentée au début de cette contribution, incite à la prudence en matière de chronologie : on a trop généreusement prêté à l'Antiquité, depuis R. Lizop (1931) jusqu'aux ouvrages les plus récents, des exploitations qui n'ont fonctionné qu'à époque plus récente, de la période

48. On recense ainsi 18 monuments à Garin, 7 à Billière, 5 à SaintAventin, 8 à Cazaril-Laspènes dans la vallée de Larboust, à l'ouest de Luchon, 4 à Vilamos dans le Val d'Aran (Espagne), 5 à Eup, 3 à BezinsGarraux, 6 à Esténos, 45 à Saint-Pé-d'Ardet dans la vallée de la Garonne. Par comparaison, les centres plus urbanisés comme Lugdunum, Onesiae (Bagnères-de-Luchon) ou, plus hypothétiquement, Saint-Béat ne comptent respectivement que 10,6 et 4 auges cinéraires ou plaques d'obturation (Laurens, 1998, carte 2). moderne à nos jours. Une carrière antique ne se cache pas systématiquement derrière chaque exploitation moderne et la caractérisation des marbres pyrénéens est encore insuffisante pour que l'on puisse, dans la plupart des cas, rattacher avec certitude à tel ou tel filon les fragments retrouvés en stratigraphie par les fouilles archéologiques des établissements urbains ou ruraux. En l'état actuel de la documentation, seules les carrières de Rapp et du Pujo de Géry, près de Saint-Béat, pour le marbre blanc, et la carrière de La Pène-Saint-Martin, pour la Brèche Isabelle, ont fourni des indices archéologiques d'exploitation antique. Pour les griottes, les carrières de L'Espiadet, près de Sainte-Marie-deCampan, certaines carrières de la vallée de la Garonne, dont les produits se retrouvent à Lugdunum, et, plus hypothétiquement, quelques exploitations du bassin du Salat, qui auraient alimenté la capitale des Consoranni, peuvent être rattachées à l'Antiquité grâce aux vestiges des sites archéologiques régionaux. Les Pyrénées n'ont donc pas de Bois des Lens et, seul, semble-t-il, le déblaiement de certains secteurs des carrières de Saint-Béat par l'exploitation actuelle est susceptible de fournir des données nouvelles dans ce domaine, illustré seulement par les découvertes de 1946 (Sapène, 1946; Fabre, Sablayrolles, 1995).

Le problème de l'identification des marbres n'en est que plus crucial, puisque c'est dans les pièces issues des fouilles archéologiques qu'il faut chercher les traces des carrières antiques. L'archéométrie, malgré des découvertes fructueuses dans le domaine des marbres blancs, comme les identifications relatives au trophée de Lugdunum ou aux sarcophages de l'église Saint-Just à Valcabrère, a révélé ses limites dans plusieurs matières. Absence d'éléments-traces suffisamment caractéristiques et variations des composantes sur un même front de carrière à quelques mètres de distance semblent rendre toute tentative hasardeuse tant dans le domaine des griottes que dans celui du marbre blanc. Les analyses, dans tous les cas de figure, ne se révèlent productives qu'au prix du croisement de plusieurs techniques, conclusion qui ressortait clairement du colloque de 1995 sur ces problèmes (Cabanot et al. éds, 1995). Le calcul statistique de la marge d'erreur, tel que C. Costedoat l'a pratiqué dans sa thèse, constitue un élément déterminant pour tester la fiabilité de l'identification, et il est regrettable qu'il ne soit pas universellement pratiqué. 
En dépit de ces difficultés, c'est en matière d'étude de la diffusion que les perspectives de travail sont les plus enrichissantes. Les inventaires récents sur les sites proches (édifices de Coupéré à Lugdunum, villae de Valentine ou Montmaurin) ou plus lointains (agglomération et environs de $\operatorname{Cos} a$ ) ont mis en lumière les inégalités dans l'approvisionnement de ces ensembles urbains ou ruraux et offrent donc matière à réflexion sur les phénomènes de commercialisation: nature des marchés, influence des modes, etc. La méthode suppose un corpus de données suffisamment important en quantité et bien cadré sur le plan géographique, chronologique et sociologique: ensemble d'une villa, ou d'un édifice urbain - parmi lesquels il faut distinguer le public du privé -, ou d'un groupe plus large, comme une agglomération secondaire ou une ville. L'attention doit être portée, dans l'analyse, aux données stratigraphiques, quand elles existent (elles seules sont susceptibles de fournir des indicateurs chronologiques), et à la rigueur la plus draconienne dans l'identification. Ce dernier point amène à grossir la proportion des « indéterminés " mais permet, seul, de donner aux hypothèses une base stable et assurée. Des recherches de ce type sont à envisager sur des ensembles plus lointains, comme la cité de Tolosa, dont la collection de marbres a été particulièrement enrichie, au cours des dernières années, par les travaux de sauvetage de l'archéologie urbaine, les villae du Gers, potentiellement riches et situées sur les vallées descendant du piémont. Les ensembles extra-régionaux mériteraient, eux aussi, une réévaluation, car, on l'a dit, il a été trop généreusement prêté à Saint-Béat et aux marbres pyrénéens. Particulièrement prometteuses, dans ce domaine, sont les découvertes des sites transpyrénéens, et notamment de Saragosse, où les placages de Saint-Béat sont nombreux, en particulier dans le revêtement du théâtre récemment fouillé ${ }^{49}$. Quel fut le circuit de ces produits? Faut-il imaginer un long transport fluvial, jusqu'à Tolosa et Narbo, par la Garonne et l'Aude avec transbordement entre Toulouse et Carcassonne, puis maritime, de Narbo à Dertosa, et, enfin, à nouveau fluvial, par l'Ebre, jusqu'à Caesaraugusta? N'est-il pas plus vraisemblable que ces pièces, si elles n'étaient pas trop pondéreuses, franchissaient à dos de mulet les Pyrénées, par les voies bien attestées des Pyrénées centrales? Comment, dans cette hypothèse, expliquer l'absence, dans la cité de Labitolosa, pendant méridional de Lugdunum près de Barbastro en Aragon, de tout marbre nord pyrénéen ? On le voit, les perspectives de recherche ne manquent pas en matière de diffusion et de commercialisation des marbres des Pyrénées centrales.

Celles-ci, sur le plan local, mettent en relief l'originalité des supports funéraires, nés de la présence des carrières et de l'adaptation à la culture romaine d'une population pyrénéenne aux traditions vivaces. C'est le même métissage des cultures qui transparaît dans l'usage des autels votifs, dont l'adoption fut, là encore, facilitée par la proximité des exploitations marbrières, à la fois producteurs économiques et vecteurs culturels comme cultuels. À ces mutations du milieu montagnard pyrénéen, dont l'importance quantitative et qualitative n'est plus à démontrer, se mesure l'impact de l'exploitation du marbre dans les vallées des Pyrénées centrales durant la période romaine, un impact d'évidence proportionnel au nombre et à la taille des exploitations comme au volume des échanges.

49. Renseignement aimablement donné, lors de la table ronde sur l'exploitation des ressources naturelles des Pyrénées (Sablayrolles dir., 2001), par Isabel Rodà Maier, invitée, avec Marcos Maier et Aureli Alvarez, à identifier les marbres du théâtre de Saragosse. 